\title{
Dysregulated autophagy contributes to caspase-dependent neuronal apoptosis
}

\author{
Yuhyun Chung ${ }^{1}$, Juhyung Lee', Shinae Jung ${ }^{1}$, Yangsin Lee ${ }^{2}$, Jin Won Cho ${ }^{1,2,3}$ and Young J. Oh ${ }^{1}$
}

\begin{abstract}
Autophagy is a regulated, intracellular degradation process that delivers unnecessary or dysfunctional cargo to the lysosome. Autophagy has been viewed as an adaptive survival response to various stresses, whereas in other cases, it promotes cell death. Therefore, both deficient and excessive autophagy may lead to cell death. In this study, we specifically attempted to explore whether and how dysregulated autophagy contributes to caspase-dependent neuronal cell death induced by the neurotoxin 6-hydroxydopamine (6-OHDA). Ultrastructural and biochemical analyses indicated that MN9D neuronal cells and primary cultures of cortical neurons challenged with 6-OHDA displayed typical features of autophagy. Cotreatment with chloroquine and monitoring autophagic flux by a tandem mRFP-EGFP-tagged LC3 probe indicated that the autophagic phenomena were primarily caused by dysregulated autophagic flux. Consequently, cotreatment with an antioxidant but not with a pan-caspase inhibitor significantly blocked 6-OHDA-stimulated dysregulated autophagy. These results indicated that 6-OHDA-induced generation of reactive oxygen species (ROS) played a critical role in triggering neuronal death by causing dysregulated autophagy and subsequent caspase-dependent apoptosis. The results of the MTT reduction, caspase-3 activation, and TUNEL assays indicated that pharmacological inhibition of autophagy using 3-methyladenine or deletion of the autophagyrelated gene Atg5 significantly inhibited 6-OHDA-induced cell death. Taken together, our results suggest that abnormal induction of autophagic flux promotes apoptotic neuronal cell death, and that the treatments limiting dysregulated autophagy may have a strong neuroprotective potential.
\end{abstract}

\section{Introduction}

Autophagy is a highly conserved cellular degradative process that involves the delivery of cytoplasmic substrates to the lysosomes ${ }^{1}$. There are three types of autophagy: macroautophagy, chaperone-mediated autophagy, and microautophagy. In macroautophagy, the targeted cytoplasmic constituents are wrapped around by the intermediary double-membrane bound vesicle called autophagosome. The autophagosome fuses with the lysosome for degradation or recycling cytoplasmic cargos. It has been recently shown that autophagy plays a wide variety of physiological and pathophysiological roles in

\footnotetext{
Correspondence: Young J. Oh (yjoh@yonsei.ac.kr)

${ }^{1}$ Department of Systems Biology, Yonsei University College of Life Science and Biotechnology, Seoul 120-749, South Korea

${ }^{2}$ Glycosylation Network Research Center, Yonsei University, Seoul 120-749,

South Korea

Full list of author information is available at the end of the article.

Edited by E. Baehrecke
}

mammalian cells $^{2,3}$. Therefore, physiological levels of autophagy must be tightly regulated because both impaired and excessive autophagy promotes cell death ${ }^{4-6}$. It has been demonstrated that autophagy plays an important role in various neurodegenerative disorders, such as Parkinson's disease (PD), Alzheimer's disease, and Huntington's disease ${ }^{7-9}$. Whether autophagy has cytoprotective $^{10-12}$ or cytotoxic ${ }^{13,14}$ effects in neurodegenerative diseases remains controversial. Intriguingly, it has been proposed that the interplay between autophagy and apoptosis may contribute to neurodegeneration ${ }^{15-17}$.

Neurotoxin-based experimental models have been used to study biochemical changes reminiscent of those occurring in patients with $\mathrm{PD}^{18}$. Among such neurotoxins, 6-hydroxydopamine (6-OHDA) has been first introduced $^{19}$. 6-OHDA is structurally similar to dopamine; it can penetrate monoaminergic neurons via dopamine and 
norepinephrine transporters and cause their death ${ }^{20}$. It has been indicated that 6-OHDA-induced toxicity is primarily ascribed to the oxidative stress generated by reactive oxygen species (ROS) and subsequent inactivation of biological macromolecules ${ }^{21}$. Numerous studies have demonstrated that 6-OHDA-treated neurons undergo apoptotic cell death ${ }^{22-24}$, whereas others have indicated that 6-OHDA treatment also induces autophagy in dopaminergic neurons ${ }^{13,25}$. Previously, we demonstrated that ROS-triggered apoptotic signaling is responsible for 6-OHDA-induced neurodegeneration ${ }^{26,27}$. Here, we attempted to address the following questions: (i) does 6-OHDA-triggered generation of ROS contribute to dysregulated autophagy? If yes, (ii) what is the potential role for ROS-induced dysregulated autophagy in the process of neuronal death? Using MN9D dopaminergic neuronal cells $^{28,29}$, mouse embryonic fibroblast (MEFs) of Atg5 knockout $(\mathrm{KO})$ cells, and primary cultures of cortical neurons exposed to 6-OHDA, we found that ROSdependent dysregulated autophagic flux contributed to capsase-3-dependent apoptosis. Intriguingly, this was quite contrary to our previous reports demonstrating that neuronal death caused by $N$-methyl-4-phenylpyridinium iodide $\left(\mathrm{MPP}^{+}\right)$was primarily accompanied by the impairment of autophagic flux, which was independent of caspase activation $^{30,31}$.

\section{Results}

\section{Autophagic events are associated with 6-OHDA-induced neurodegeneration}

To characterize 6-OHDA-induced autophagy in MN9D cells, we first examined ultrastructural changes associated with 6-OHDA-induced neurodegeneration. Using previously described approaches for ultrastructural characterization of autophagy ${ }^{32-34}$, we detected more autophagic vacuoles, including double-membraned autolysosomes, in MN9D cells treated with 6-OHDA compared to nontreated control cells (Fig. 1a). On closer inspection, we found that autophagic vacuoles were scattered around the cytosol (Fig. 1b; black arrows). Phagophores also were detected in the cytosol (Fig. 1b; white arrow). Enlarged electron microscopic images of autophagic vacuoles typically detected in 6-OHDAtreated cells were shown (Fig. S1). After analyzing ten randomly selected cells (ten serial ultrathin sections per cell), we found that the number of autophagic vacuoles was markedly increased after 6-OHDA treatment (Fig. 1c). In a separate study, immunoblot analyses were performed to measure the expression levels of Rab5 and Rab7 as markers of early endosome and late endosome/ multivesicular bodies, respectively. No discernible changes in the expression levels of Rab5 and Rab7 were found in MN9D cells following 6-OHDA treatment (Fig. S2a). Immunocytochemistry also revealed that there were no obvious signs of colocalization of LC3 with either Rab5 or Rab7 in MN9D cells in either 6-OHDA-treated or 6-OHDA-nontreated cells (Fig. S2b, c).

Microtubule-associated protein light chain 3 (MAP1LC3/LC3) takes part in autophagosome biogenesis and substrate selection ${ }^{32,33,35,36}$. Cytoplasmic LC3-I is formed following Atg4-mediated C-terminal cleavage and conjugates with phosphatidylethanolamine to form lipidated LC3-II. LC3-II levels and LC3 puncta correlate with the number of autophagosomes. Therefore, we performed biochemical analyses of 6-OHDA-treated MN9D cells using an anti-LC3 antibody. LC3-II immunoreactivity appeared as early as $6 \mathrm{~h}$ after 6-OHDA treatment and progressively increased thereafter (Fig. 2a). Quantitative analyses showed that the relative levels of LC3-II increased during the incubation with 6-OHDA (Fig. 2b). This increase was parallel to the activation of caspase-3. Consistently, immunofluorescence analyses revealed that LC3 punctate staining was enhanced in 6-OHDA-treated MN9D cells compared to that in nontreated control cells (Fig. 2c-e). Taken together, our results indicated that morphological and biochemical changes typical of autophagy accompanied 6-OHDA-induced dopaminergic neurodegeneration.

\section{6-OHDA-triggered ROS contributes to autophagic events that precedes caspase-dependent apoptosis}

Previously, numerous studies demonstrated that ROSmediated apoptotic signaling increased within several hours after 6-OHDA treatment ${ }^{21,24,37,38}$ and caused neuronal cell death ${ }^{22-24,39,40}$. Therefore, we investigated whether an antioxidant or a caspase inhibitor could affect 6-OHDA-induced autophagy. First, we treated MN9D cells with 6-OHDA in the presence or absence of $\mathrm{N}$-acetyl-L-cysteine (NAC), a widely used antioxidant. As we previously demonstrated ${ }^{22}$, typical 6-OHDA-induced apoptotic morphological features such as shrinkage or condensation of the cytoplasmic membrane, were partially rescued following cotreatment with NAC (Fig. 3a). Interestingly, NAC cotreatment reduced not only druginduced cleaved caspase-3 levels but also LC3-II levels (Fig. 3b-d). Similarly, immunocytochemical localization analyses revealed that 6-OHDA-induced increase in the number and area of LC3 dots was markedly reduced in the presence of NAC (Fig. 3e-g), indicating that ROS were responsible for autophagic events during 6-OHDAinduced cell death. We then used the pan-caspase inhibitor $N$-benzyloxycarbonyl-Val-Ala-Asp-fluoromethylketone (Z-VAD-FMK) to examine whether ROS-induced activation of caspase would affect 6-OHDA-induced autophagy. Cotreatment with Z-VAD-FMK prevented 6-OHDA-induced morphological changes and caspase-3 activation (Fig. 3h-k). However, Z-VAD-FMK had little effect on the LC3-II level, and number and area of LC3 
a

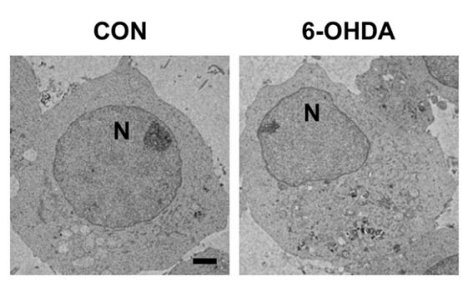

C

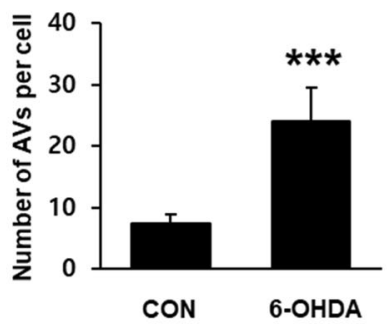

b
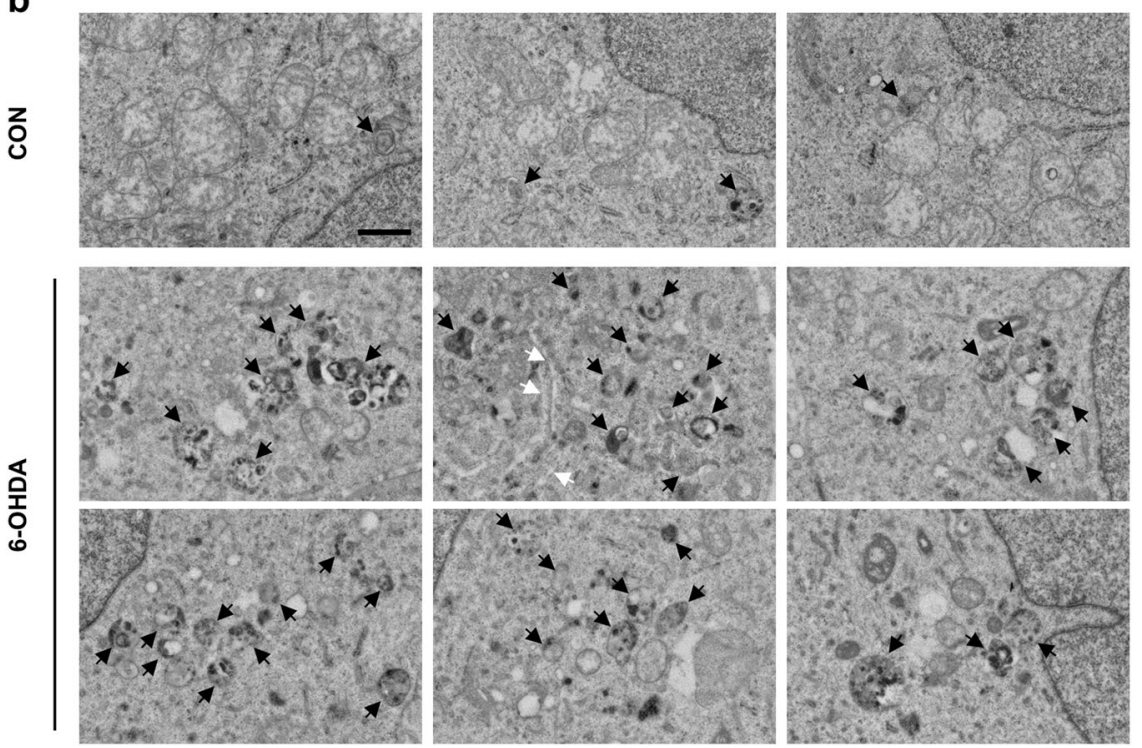

Fig. 1 Ultrastructural features of autophagy induced by 6-OHDA in MN9D cells. a Electron micrographs were taken after treatment with or without $100 \mu \mathrm{M}$ 6-OHDA for $15 \mathrm{~h}$. N represents nucleus. Scale bar represents $2 \mu \mathrm{m}$. b Enlarged images of 6-OHDA-treated cells illustrate typical of autophagic vacuoles (AVs; black arrows) and phagophore (white arrows). Scale bar represents $1 \mu \mathrm{m}$. c The number of AVs was quantified from ten randomly selected cells per each group. Bars represent the mean \pm standard deviation ( $7.4 \pm 1.4$ for untreated control vs. $24 \pm 5.5$ for 6-OHDA-treated cells). ${ }^{* * *} P<0.001$

dots (Fig. 3i-n), indicating that autophagic events preceded 6-OHDA-induced apoptosis.

\section{6-OHDA treatment causes dysregulated autophagic flux}

Autophagic flux is a sequence of events in the autophagic pathway starting from the autophagosome formation to the degradation of autophagic substrates ${ }^{41}$. Accumulation of autophagosomes and increased levels of LC3-II indicate either activation of autophagic induction or blockade of downstream autophagic degradation steps $^{1,33}$. To determine whether 6-OHDA-mediated accumulation of LC3-II and increase in LC3 dots are due to the activation of autophagy or blockade of lysosomal degradation, we monitored autophagic flux after cotreatment with the lysosomal inhibitor chloroquine $(\mathrm{CQ})^{32,42}$. Immunoblot analyses indicated that LC3-II levels were higher in MN9D cells cotreated with 6-OHDA and CQ than in MN9D cells treated with 6-OHDA alone
(Fig. 4a, b). The levels of p62/sequestosome-1 (p62), a well-known autophagic cargo protein ${ }^{32}$ were decreased in MN9D cells following 6-OHDA treatment and restored in CQ cotreated cells (Fig. 4a-c), indicating that p62 is rapidly degraded during 6-OHDA-induced autophagy. As demonstrated by others ${ }^{15,16}$, the restoration level of p62 in CQ cotreated cells was less than that in cells treated with $\mathrm{CQ}$ alone. No obvious difference in the levels of caspase activation was detected in cells treated with 6-OHDA alone or in combination with CQ (Fig. 4a-d). Unlike the previous report, which demonstrates caspase-dependent p62 cleavage ${ }^{43}$, no signs of p62 cleavage were detected in 6-OHDA-treated or 6-OHDA-nontreated cells (Fig. S3). Quantitative analyses demonstrated that the number and area of LC3 dots per cell were increased in MN9D cells cotreated with CQ compared to those in MN9D cells treated with 6-OHDA alone (Fig. 4e-g). To further support our hypothesis, we monitored autophagic flux using a 


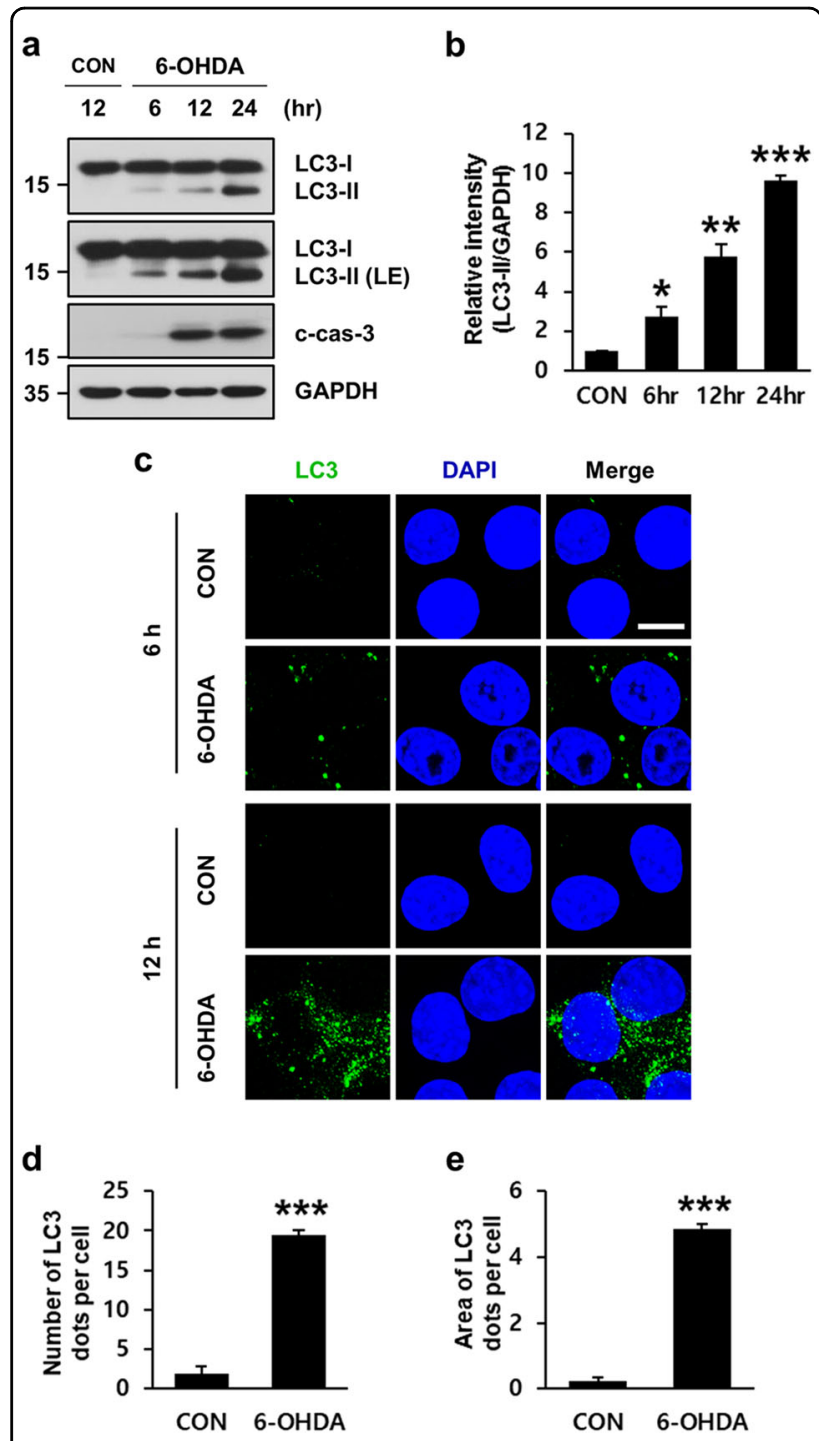

Fig. 2 Biochemical characterization of autophagy induced by 6-OHDA in MN9D cells. a Cells were treated with or without $100 \mu \mathrm{M}$ 6-OHDA for the indicated time periods. Immunoblot analyses were performed using anti-LC3 and anti-cleaved caspase-3 (c-cas-3) antibodies. Anti-GAPDH antibody was utilized as loading control. b The intensity of LC3-II signals at each timepoint was

densitometrically measured using ImageJ, normalized by the intensity of GAPDH signal, and expressed as fold change relative to the untreated control value. Bars represent the mean \pm standard deviation of three independent experiments $(6 h, 2.7 \pm 0.5 ; 12 h, 5.8 \pm 0.6 ; 24 h$, $9.7 \pm 0.2) .{ }^{*} P<0.05 ;{ }^{* *} P<0.01$; ${ }^{* *} P<0.001$. c Cells treated with or without $100 \mu \mathrm{M} 6-\mathrm{OHDA}$ for the indicated time periods were subjected to immunocytochemical analyses using an anti-LC3 antibody (green) and nuclei counterstaining with Hoechst 33258 (blue). Cells were then examined under a confocal microscope. Merged images are provided in the right panels. Scale bars represent $10 \mu \mathrm{m}$. d, e The number (d) and area (e) of LC3 dots per cell were quantified using ImageJ in $100 \mu \mathrm{M}$ 6-OHDA-treated cells for $12 \mathrm{~h}$. Data are shown as the mean \pm standard deviation of three independent experiments (number of LC3 dots, $2.0 \pm 0.9$ for control vs. $19.4 \pm 0.7$ for 6-OHDA-treated cell; area of LC3 dots, $0.2 \pm 0.1$ for control vs. $4.9 \pm 0.1$ for 6-OHDA-treated cell). ${ }^{* * *} P<0.001$ tandem mRFP-EGFP-tagged LC3 probe ${ }^{32,33}$. Immunofluorescence and quantitative analyses of mRFP-EGFPLC3 indicated that the number of autophagosomes (yellow dots, $\mathrm{EGFP}^{+} / \mathrm{mRFP}^{+}$) and autolysosomes (red dots, $\mathrm{EGFP}^{-} / \mathrm{mRFP}^{+}$) were increased following 6-OHDA treatment (Fig. 4h, i). Taken together, these data indicated that 6-OHDA treatment caused dysregulated autophagic flux in MN9D cells.

To obtain additional evidence of the dysregulated induction of autophagy in 6-OHDA-treated MN9D cells, we determined expression levels of the autophagic signaling molecules. Mammalian target of rapamycin (mTOR) is a master regulator of autophagy and phosphorylates p70S6K. Akt phosphorylates and thereby activates mTOR to inhibit autophagy, whereas AMPactivated protein kinase (AMPK), which is a key energy sensor and that regulates cellular metabolism to maintain energy homeostasis, promotes autophagy ${ }^{44}$. The activity of mTOR and Akt decreased following exposure to 6OHDA as demonstrated by lower intensities of p-mTOR, p-p70S6K, and p-Akt bands, respectively (Fig. $4 \mathbf{j}-\mathrm{n})$. The levels of p-AMPK, the active form of AMPK, markedly increased following 6-OHDA treatment (Fig. 4j-n), supporting our hypothesis that 6-OHDA induced autophagic signaling. To determine whether the drug caused any changes in lysosomal activity, the cells treated with or without 6-OHDA were stained with LysoTracker Red. No significant difference was observed between fluorescent emissions of 6-OHDA-treated and control cells (Fig. 4o, p). The expression pattern of the lysosomal hydrolase cathepsin D after 6-OHDA treatment was similar to that observed in control cells (Fig. 4q), implying that lysosomal function remained relatively intact. Taken together, our results demonstrate that 6-OHDA-mediated accumulation of autophagosomes was caused by dysregulated de novo formation of autophagosomes. Intriguingly, this mechanism is quite contrary to the effect of other neurotoxins (such as $\mathrm{MPP}^{+}$or nigericin), which led to LC3-II accumulation primarily due to impaired autophagic flux ${ }^{30,31}$.

\section{Inhibition of autophagy induction prevents 6-OHDA- induced apoptosis}

To evaluate the functional role of dysregulated autophagic induction in 6-OHDA-triggered neuronal cell death, MN9D cells were incubated with 3-methyladenine (3-MA), a cell-permeable inhibitor of type III phosphatidylinositol 3kinase (PI3K) that blocks autophagosome formation ${ }^{45}$. Blockade of autophagic induction by cotreatment with 3-MA prevented 6-OHDA-induced cell shrinkage, so that cotreated cells had morphological features quite identical to those of cells treated with 3-MA alone or nontreated control group (Fig. 5a). We then conducted the 3-(4,5-dimethylthiazol-2-yl)-2,5-diphenyltetrazolium bromide (MTT) 


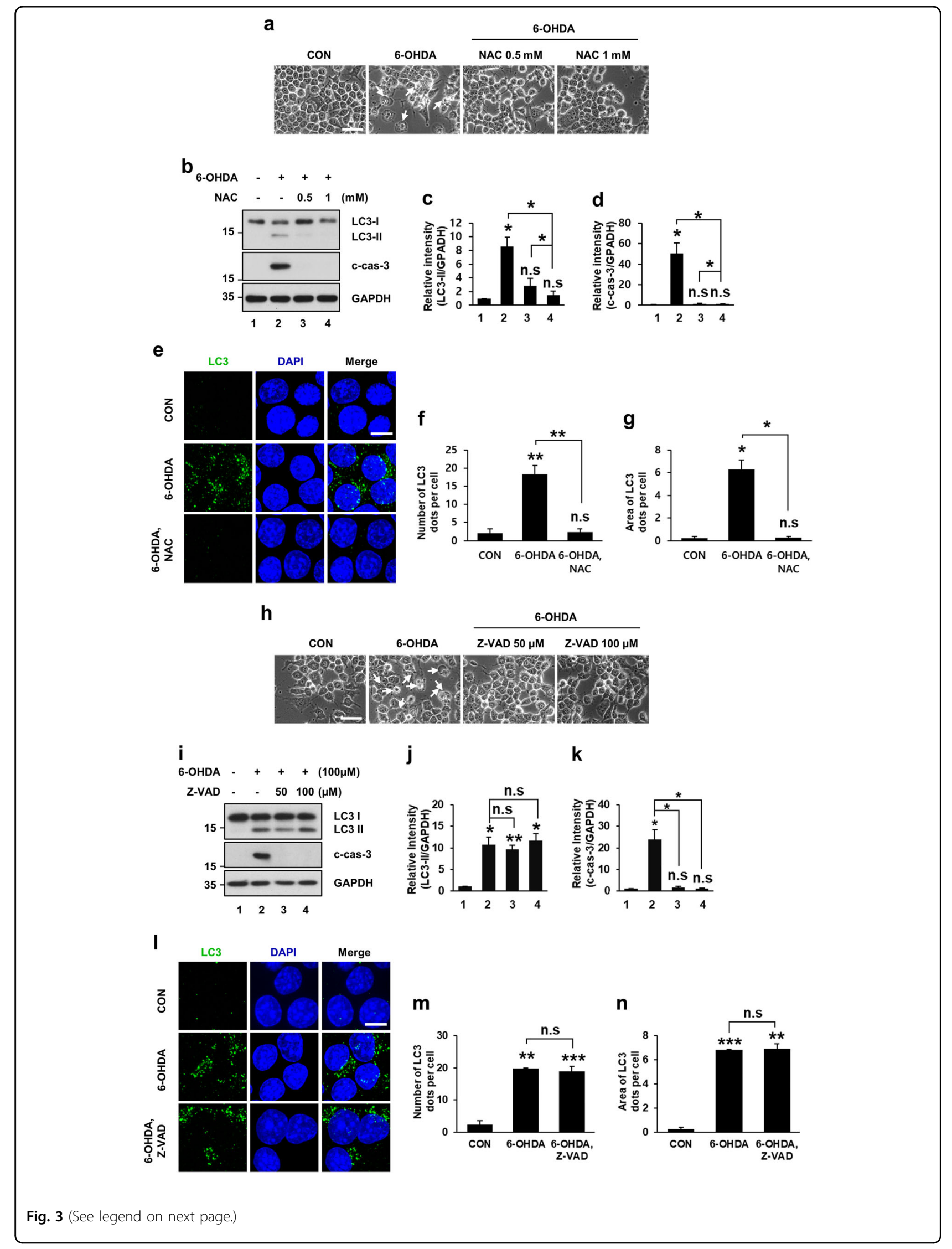


(see figure on previous page)

Fig. 3 6-OHDA-triggered autophagy and cell death in MN9D cells is prevented by an antioxidant but not by a pan-caspase inhibitor. Cells were treated with $100 \mu \mathrm{M} 6-\mathrm{OHDA}$ for $12 \mathrm{~h}$ in the presence or absence of the indicated concentrations of the antioxidant $\mathrm{N}$-acetyl-L-Cysteine (NAC) $(\mathbf{a}-\mathbf{g})$ or the pan-caspase inhibitor Z-VAD-FMK (Z-VAD) $(\mathbf{h}-\mathbf{n}) . \mathbf{a}, \mathbf{h}$ Cells were then examined by a phase-contrast microscopy. Dying cells were indicated by white arrows. Scale bar represents $10 \mu \mathrm{m}$. b, $\mathbf{i}$ Immunoblot analyses were performed using anti-LC3 and anti-cleaved caspase-3 antibody (c-cas-3). $\mathbf{c}, \mathbf{d}, \mathbf{j}, \mathbf{k}$ After normalization by GAPDH signal intensity, the relative intensity of LC3-II and c-cas-3 signals was expressed as a fold change relative to untreated control value. Bars represent the mean \pm standard deviation of three independent experiments (LC3- $I$, c $8.6 \pm 1.4$ for 6-OHDAtreated vs. $2.9 \pm 1.0$ for 6-OHDA plus $0.5 \mathrm{mM}$ NAC-treated vs. $1.5 \pm 0.5$ for 6-OHDA-treated plus $1 \mathrm{mM}$ NAC-treated cells; $\mathbf{j}$ 10.8 \pm 1.7 for 6 -OHDAtreated vs. $9.6 \pm 0.97$ for 6-OHDA plus $50 \mu \mathrm{M}$ Z-VAD-FMK-treated vs. $11.7 \pm 1.6$ for 6-OHDA plus $100 \mu \mathrm{M}$ Z-VAD-FMK-treated cells; c-cas-3, d $50.5 \pm 10.2$ for 6-OHDA-treated vs. $1.5 \pm 0.5$ for 6-OHDA plus $0.5 \mathrm{mM} \mathrm{NAC-treated} \mathrm{vs.} 1.4 \pm 0.1$ for 6-OHDA plus $1 \mathrm{mM}$ NAC-treated cells; $\mathbf{k} 24.0 \pm 4.5$ for $6-\mathrm{OHDA}$ treated vs. $1.6 \pm 0.37$ for 6-OHDA plus $50 \mu \mathrm{M}$ Z-VAD-FMK-treated vs. $1.0 \pm 0.3$ for $6-O H D A$ plus $100 \mu \mathrm{M} Z$-VAD-FMK-treated cells). ${ }^{*} P<0.05$; ${ }^{* *} P<0.01$; n.s. not significant. e, I After staining with an anti-LC3 antibody (green), confocal immunofluorescent images of LC3 were taken. Nuclei were counterstained with Hoechst 33258 (blue). Scale bar represents $10 \mu \mathrm{m} . \mathbf{f}, \mathbf{g}, \mathbf{m}, \mathbf{n}$ The numbers of LC3 dots (f, $\mathbf{m})$ and LC3 dot area (g, $\mathbf{n})$ were then quantified using ImageJ. Bars represent the mean \pm standard deviation of three independent experiments (number of LC3 dots, $\mathbf{f} 2.1 \pm 1.2$ for control vs. $18.4 \pm 2.3$ for 6-OHDA-treated vs. $2.4 \pm 0.9$ for 6-OHDA plus $1 \mathrm{mM} \mathrm{NAC}$-treated cells; $\mathbf{m} 2.4 \pm 1.1$ for control vs. $19.8 \pm 0.3$ for $6-\mathrm{OHDA}$-treated vs. $19.0 \pm 1.6$ for 6-OHDA plus $100 \mu \mathrm{M}$ Z-VAD-FMK-treated cells; area of LC3 dots; $\mathbf{g} 0.2 \pm 0.1$ for control vs. $6.3 \pm 0.8$ for 6 -OHDA-treated vs. $0.3 \pm 0.1$ for 6-OHDA plus NAC-treated cells; $\mathbf{n} 0.3 \pm 0.1$ for control vs. $6.8 \pm 0.1$ for 6 -OHDA-treated vs. $6.9 \pm 0.4$ for 6-OHDA plus Z-VAD-FMK-treated cells). ${ }^{*} P<0.05 ;{ }^{* * P}<0.01 ;{ }^{* * *} P<0.001$; n.s. not significant

reduction assays to determine whether inhibition by 3-MA of 6-OHDA-induced autophagic induction would affect cell death. We observed that MN9D cells cotreated with 3-MA showed increased resistance against 6-OHDA-induced toxicity (Fig. 5b).

To elucidate the mechanisms by which 3-MA prevented 6-OHDA-mediated cell death, we determined whether there was interplay between autophagy induction and caspase-3 activation. LC3-II levels in the group cotreated with 3-MA were markedly reduced (Fig. 5c, d). In contrast, 6-OHDA-induced decreases in p62 levels were partially recovered by cotreatment with 3-MA (Fig. 5c-e). Interestingly, 3-MA cotreatment also prevented 6-OHDA-triggered caspase-3 activation (Fig. 5c-f). We next inquired whether 3-MA cotreatment also blocked the release of cytochrome $c$ to the cytosol, an event that triggers the onset of apoptosis by activating caspases ${ }^{46-48}$. Double immunofluorescence staining revealed that cytochrome $c$ was colocalized with mitochondrial import receptor subunit TOM20 in untreated control cells (Fig. 5g, upper panel). Upon exposure to 6-OHDA, cytochrome $c$ staining became diffused and not colocalized with TOM20 (Fig. 5g, middle panel), indicating that 6-OHDA treatment caused the release of cytochrome $c$ to the cytosol. In contrast, cotreatment with 3-MA resulted in cytochrome $c$ staining pattern quite similar to that observed in nontreated control cells (Fig. 5g, lower panels). More specifically, the quantification analyses revealed that the percentage of colocalization between cytochrome $c$ and TOM20 over the total area of TOM20 was markedly decreased following 6-OHDA treatment but significantly restored by cotreatment with 3-MA (Fig. 5h). To further confirm whether 6-OHDA-induced dysregulated autophagic induction is linked to apoptotic cell death, TUNEL staining was conducted in MN9D cells treated with 6-OHDA in the presence or absence of 3-MA. The number of TUNEL-positive cells was dramatically increased after 6-OHDA treatment (Fig. 5i). Autophagic inhibition by cotreatment with 3-MA reduced the percentage of TUNEL-positive cells by approximately $30 \%$ (Fig. 5j). From these data, we hypothesized that 6-OHDA-induced dysregulated autophagic induction promoted caspase-3-dependent neuronal cell death.

Because pharmacological effects might be insufficiently specific, to strengthen out hypothesis, we used $\operatorname{Atg} 5$ knockout (KO) MEFs in which autophagy cannot proceed due to the lack of Atg $5{ }^{44}$. First, we measured cell viability by the MTT reduction assay to determine whether 6-OHDA-induced cell death was prevented in Atg5 KO MEFs. As shown in Fig. 6, we found that Atg5 knockout itself did not affect cell viability. Compared with the viability of wild-type (WT) MEFs, deletion of Atg5 partially protected against 6-OHDA-induced cell death (Fig. 6a). The phenotype rescue was even more prominent when expression levels of LC3-II, p62, and activated caspase-3 were compared in WT and Atg5 KO MEFs. Immunoblot analyses revealed that 6-OHDA-induced accumulation of LC3-II, reduction in p62, and activation of caspase-3 were significantly blocked in Atg5 KO MEFs (Fig. 6b-e). As determined by colocalization between cytochrome $c$ and TOM20, we found that the 6-OHDA-induced release of cytochrome $c$ to the cytosol in WT MEFs also was inhibited in Atg5 KO MEFs (Fig. 6f, g). In support of these data, we found that the number of TUNEL-positive nuclei in WT MEFs following 6-OHDA treatment was significantly reduced in 6-OHDA-treated Atg5 KO MEFs (Fig. 6h, i). Thus, both pharmacological and genetic experiments demonstrated that dysregulated autophagy induced by 6-OHDA contributed to caspase-3-dependent apoptosis. 


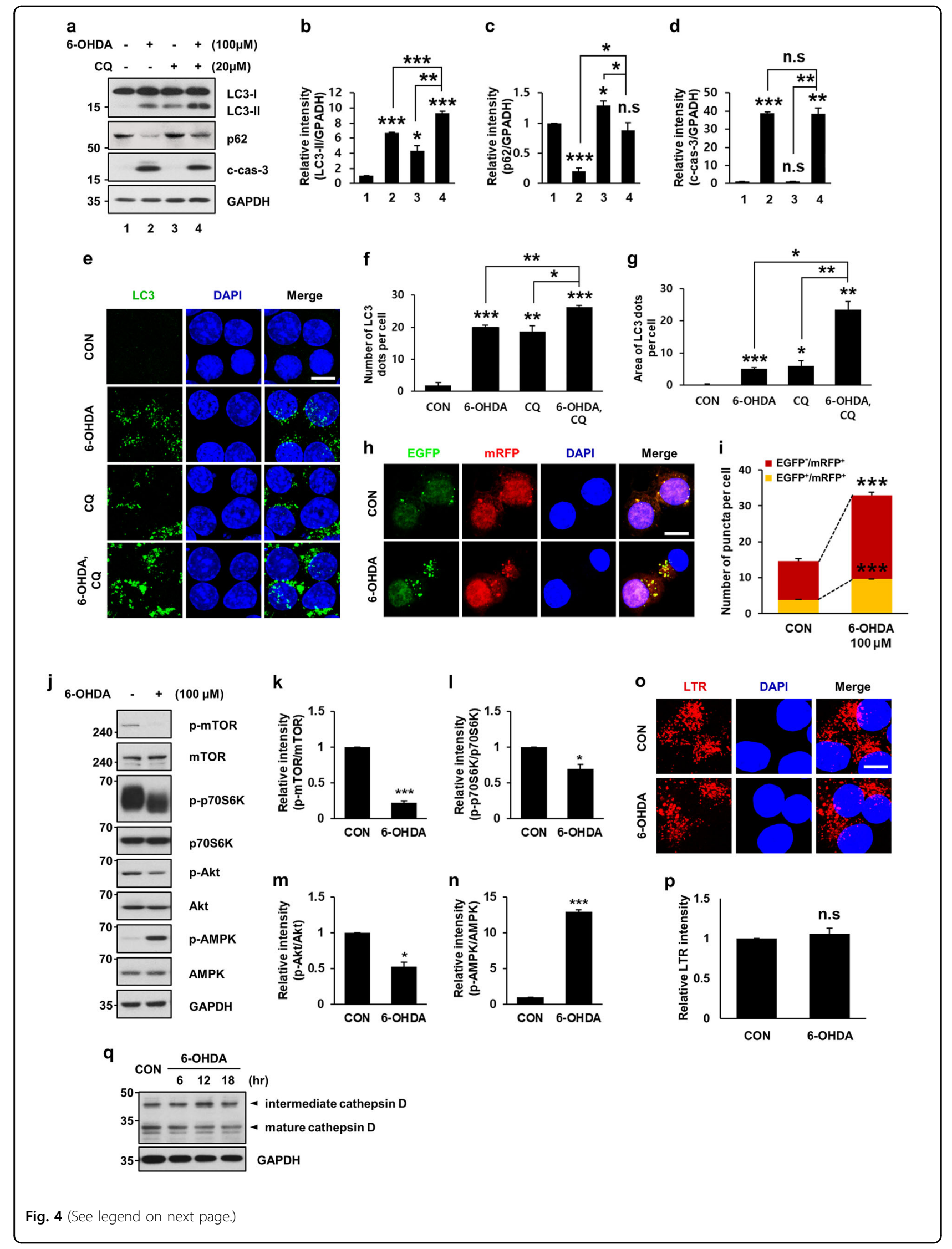


(see figure on previous page)

Fig. 4 Dysregulated autophagy induced by 6-OHDA treatment. MN9D cells were incubated with $100 \mu \mathrm{M} 6-\mathrm{OHDA}$ for $12 \mathrm{~h}$ in the presence or absence of $20 \mu \mathrm{M}$ chloroquine (CQ). a Autophagic flux in lysates was monitored and compared after immunoblotting with an anti-LC3 and anti-p62 antibody. Activation of caspase-3 was also measured using an anti-c-cas-3 antibody. $\mathbf{b}-\mathbf{d}$ After normalization by GAPDH signal intensity, the relative intensity of LC3-II, p62, and c-cas-3 signals from each treatment was expressed as a fold change relative to untreated control value. Bars represent the mean \pm standard deviation of three independent experiments (LC3-II, $6.7 \pm 0.1$ for 6-OHDA-treated vs. $4.4 \pm 0.6$ for CQ-treated vs. 9.3 \pm 0.2 for 6-OHDA plus CQ-treated cells; p62, $0.2 \pm 0.1$ for 6-OHDA-treated vs. $1.3 \pm 0.1$ for CQ-treated vs. $0.9 \pm 0.1$ for 6 -OHDA plus CQ-treated cells; c-cas-3, $38.7 \pm 1.0$ for 6-OHDA-treated vs. $1.1 \pm 0.1$ for CQ-treated vs. $38.5 \pm 3.1$ for 6 -OHDA plus CQ-treated group). ${ }^{*} P<0.05 ;{ }^{* *} P<0.01$; ${ }^{* * *} P<0.001$; n.s. not significant. e Immunocytochemical localization of LC3 puncta was carried out following treatment with $100 \mu \mathrm{M} 6-\mathrm{OHDA}$ for $12 \mathrm{~h}$ in the presence or absence of CQ. Confocal immunofluorescence images represent LC3 dots (green) and nuclei (blue). Scale bar represents $10 \mu \mathrm{m}$. $\mathbf{f}, \mathbf{g}$ The number (f) and area (g) of LC3 dots per cell were quantified using ImageJ. Bars represent the mean \pm standard deviation of three independent experiments (number of LC3 dots, $1.9 \pm 0.9$ for control vs. $20.0 \pm 0.8$ for 6-OHDA-treated vs. $18.7 \pm 1.8$ for CQ-treated vs. $26.2 \pm 0.5$ for 6-OHDA plus CQ-treated cells; area of LC3 dots, $0.2 \pm 0.1$ for control vs. $5.1 \pm 0.3$ for 6 -OHDA-treated vs. $6.0 \pm 1.6$ for CQ-treated vs. $23.4 \pm 2.6$ for 6 -OHDA plus CQ-treated cells). ${ }^{*} P<0.05$; ${ }^{* *} P<0.01 ;{ }^{* *} P<0.001$. h MN9D cells were transiently transfected with the mRFP-EGFP-LC3 plasmid for $48 \mathrm{~h}$ and then treated with $100 \mu \mathrm{M} 6-\mathrm{OHDA}$ for $12 \mathrm{~h}$. Confocal immunofluorescence images represent EGFP dots (green), mRFP dots (red), and nuclei (blue). Merged images are provided in the right panels. Scale bar represents $10 \mu \mathrm{m}$. i The number of puncta per cell that were EGFP ${ }^{+} / \mathrm{mRFP}^{+}$or $\mathrm{EGFP}^{-} / \mathrm{mRFP}^{+}$were quantified using ImageJ. Bars represent the mean \pm standard deviation of three independent experiments (EGFP ${ }^{+} / \mathrm{mRFP}^{+}, 3.8 \pm 0.1$ for control vs. 9.6 \pm 0.1 for 6-OHDA-treated cells; EGFP ${ }^{-} / \mathrm{mRFP}^{+}, 10.8 \pm 0.7$ for control vs. $23.4 \pm 0.8$ for 6 -OHDA-treated cells). ${ }^{* *} P<0.001$. j Following treatment with $100 \mu \mathrm{M} 6-\mathrm{OHDA}$ for $12 \mathrm{~h}$, cellular lysates were blotted with antibodies against autophagy signaling molecules. $\mathbf{k}-\mathbf{n}$ After normalization against intensity of total protein, the fold intensity of the phosphorylated forms was expressed as a fold change relative to untreated control value. Bars represent the mean \pm standard deviation of three independent experiments $\left(\mathbf{k} 0.2 \pm 0.03 ; \mathbf{I} 0.7 \pm 0.1 ; \mathbf{m ~} 0.5 \pm 0.1 ; \mathbf{n} 13.0 \pm 0.2\right.$ for 6 -OHDA-treated cells). ${ }^{*} P<0.05 ;{ }^{* * *} P<0.001 . \mathbf{o}$ To assess lysosomal activity, MN9D cells exposed to $100 \mu \mathrm{M}$ 6-OHDA for $12 \mathrm{~h}$ were stained with $0.5 \mu \mathrm{M}$ LysoTracker ${ }^{\circledast}$ Red DND-99 (LTR) and subjected to confocal microscopy. Cell nuclei were counterstained with Hoechst 33258 (blue). Scale bar represents $10 \mu \mathrm{m}$. $\mathbf{p}$ LTR intensity was quantified from at least 100 randomly selected cells using ImageJ software, and expressed as the fold change relative to the untreated control value. Bar represents the mean \pm standard deviation of three independent experiments (1.1 \pm 1.3 for 6-OHDA-treated cells). n.s. not significant. q Following treatment with $100 \mu \mathrm{M} 6-\mathrm{OHDA}$ for the indicated time periods, expression of various forms of the lysosomal hydrolase cathepsin D was immunoprobed with an anticathepsin D antibody

\section{6-OHDA-induced increase in autophagy promotes death of} mouse primary cortical neurons

Having established the sequence of events between dysregulated autophagic induction and apoptosis in MN9D cells and MEFs, we sought to translate these findings to primary cultures of cortical neurons. Following incubation with 6-OHDA, LC3-II levels increased and p62 levels decreased concomitantly with caspase- 3 activation (Fig. 7a-d). Cotreatment of cortical neurons with CQ accelerated LC3-II accumulation and partially restored 6-OHDA-induced p62 degradation (Fig. 7a-c). No significant difference in caspase activation was detected in cortical neurons treated with 6-OHDA alone or in combination with CQ (Fig. 7a-d). These results indicated that autophagy induction after 6-OHDA treatment was also prominent in primary cultures of cortical neurons.

To investigate whether 6-OHDA-induced neuronal cell death was attributable to dysregulated autophagy, 3-MA was used to block autophagy in cortical neurons treated with 6-OHDA. A phase-contrast microscopy demonstrated that cell shrinkage caused by 6-OHDA was blocked in the presence of 3-MA (Fig. 7e). MTT reduction assays also revealed that 6-OHDA-mediated neuronal death was significantly blocked in the presence of 3-MA (Fig. 7f). We then analyzed whether neuronal protection by 3-MA was a consequence of inhibition of 6-OHDAinduced dysregulated autophagy and caspase-3 activation. Immunoblot analyses indicated that 6-OHDA-induced increase in LC3-II and cleaved caspase-3 was attenuated in the presence of 3-MA (Fig. 7g-j). In cortical neurons cotreated with 3-MA, 6-OHDA-induced decreases in p62 were restored to nontreated control levels (Fig. 7i). The TUNEL assay also indicated that 6-OHDA-induced DNA fragmentation was markedly inhibited in cortical neurons cotreated with 3-MA (Fig. 7k, 1). In summary, our data provide evidence for a cytotoxic role of dysregulated autophagic induction in neuronal cells caused by the activation of downstream caspase-dependent pathway.

\section{Discussion}

Here, we demonstrated that exposure of MN9D cells, MEFs, and primary cortical neurons to 6-OHDA caused dysregulated autophagic induction and subsequent caspase-dependent apoptosis. Inhibition of autophagy by pharmacological and genetic means protected against 6OHDA-induced cell death. Our present data are consistent with recent study by $\mathrm{He}$ et al. ${ }^{13}$, which also reported on the role of 6-OHDA-induced autophagy in neurotoxicity. The findings from this study and our study are in contrast to other reports ${ }^{49}$, which have shown that 6-OHDA induces autophagic flux dysfunction by impairing transcription factor EB activation and lysosomal function in dopaminergic neurons and SH-SY5Y cells. Although this discrepancy is not yet understood, our data-which was acquired from various cell types exposed to 6-OHDA-clearly demonstrate a temporal sequence of cell death that starts with autophagy induction and is followed by activation of apoptosis. Schweichel and 


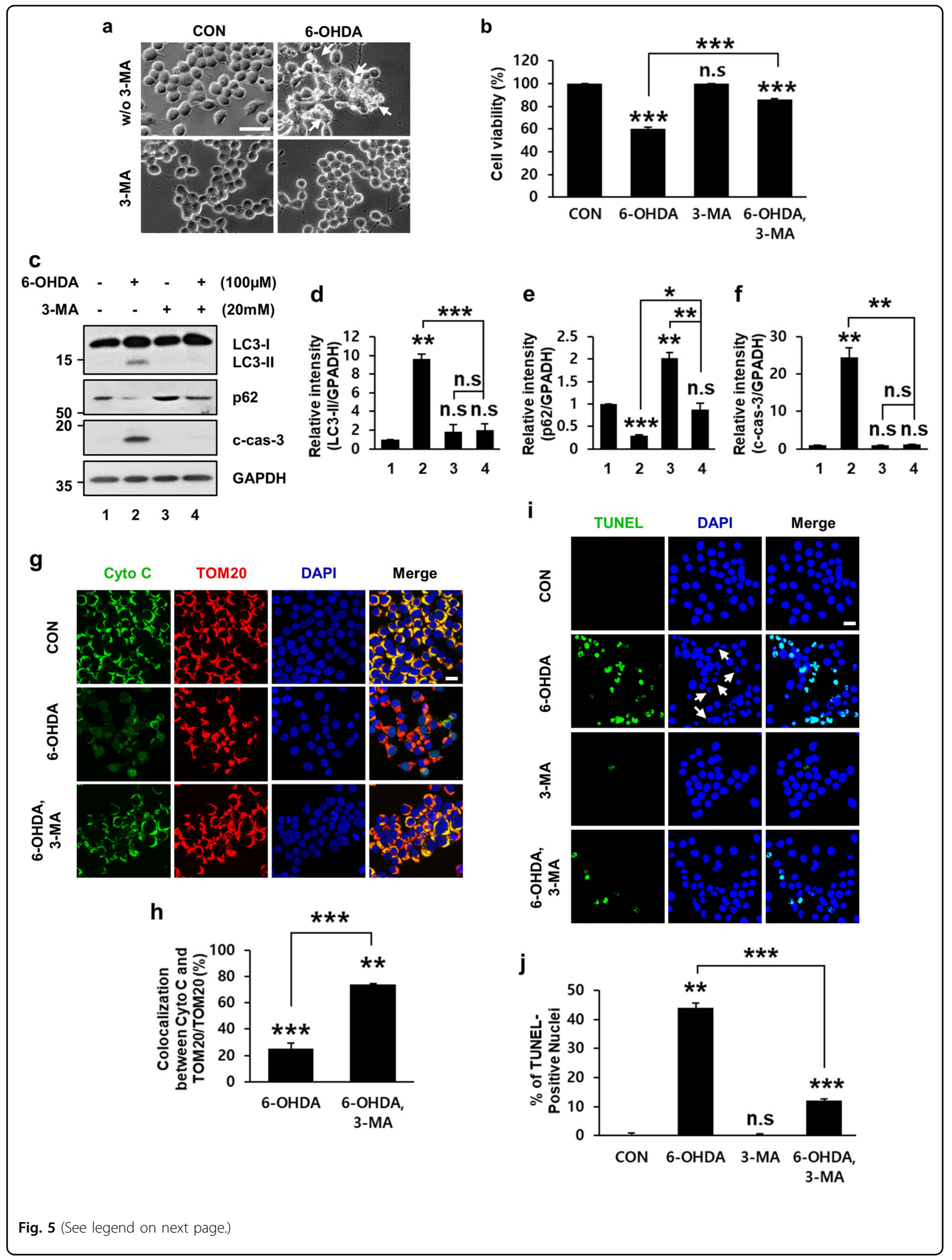


(see figure on previous page)

Fig. 5 Rescue of 6-OHDA-induced neurodegeneration by 3-metyladenine (3-MA). MN9D cells were treated for $12 \mathrm{~h}$ with or without $100 \mu \mathrm{M}$ 6OHDA or $20 \mathrm{mM}$ 3-MA alone or in combination. a Cells were analyzed by phase-contrast microscopy. Shrunken, phase-bright cells are indicated by white arrows. Scale bar represents $100 \mu \mathrm{m}$. $\mathbf{b}$ MTT reduction assays were performed to assess cell viability expressed as percentage over the untreated control cells (100\%; 60.4 $\pm 1.3 \%$ for 6-OHDA-treated vs. $85.8 \pm 0.8 \%$ for 6-OHDA plus 3-MA-treated cells). Bars represent the mean \pm standard deviation of three independent experiments. ${ }^{* *} P<0.001$; n.s. not significant. c Cell lysates were subjected to immunoblotting using anti-LC3, anti-p62 and anti-c-cas-3 antibodies. $\mathbf{d}-\mathbf{f}$ After normalization against GAPDH, densitometric values of LC3-II, p62, and c-cas-3 signals were expressed as a fold change relative to untreated control value. Bars represent the mean \pm standard deviation of three independent experiments (LC3-II, 9.6 \pm 0.5 for 6OHDA-treated vs. $1.9 \pm 0.7$ for 3-MA-treated vs. $2.0 \pm 0.7$ for 6-OHDA plus 3-MA-treated cells; p62, $0.3 \pm 0.01$ for 6-OHDA-treated vs. $2.0 \pm 0.1$ for 3-MAtreated vs. $0.9 \pm 0.1$ for 6-OHDA plus 3-MA-treated cells; c-cas-3, $24.6 \pm 2.5$ for 6-OHDA-treated vs. $0.9 \pm 0.04$ for 3-MA-treated vs. $1.1 \pm 0.2$ for 6-OHDA plus 3-MA-treated cells). ${ }^{*} P<0.05 ;{ }^{* *} P<0.01$; ${ }^{* *} P<0.001$; n.s. not significant. g, i Confocal images of cytochrome $c$ (cyto $c$, green), TOM20 (red) and TUNEL staining (green) in MN9D cells treated with $100 \mu \mathrm{M}$ 6-OHDA alone or in combination with $20 \mathrm{mM}$ 3-MA were shown. Nuclei were

counterstained with Hoechst 33258 (blue). Merged images are shown in the right panel. White arrows indicate condensed or fragmented nuclei. Scale bar represents $20 \mu \mathrm{m}$. $\mathbf{h}$ The percentage of colocalization between cyto $\mathrm{c}$ and TOM20 over total TOM20 was expressed over the untreated control cells (100\%). Confocal images of at least 30 randomly selected cells from each of the three independent experiments we used for quantitation. Bars represent the mean \pm standard deviation of three independent experiments $(25.4 \pm 1.1 \%$ for 6-OHDA-treated vs. $74.1 \pm 0.9 \%$ for 6 OHDA plus 3-MA-treated group). ${ }^{*} P<0.01 ;{ }^{* *} P<0.001$. $\mathbf{j}$ The percentage of TUNEL-positive nuclei was assessed from at least 100 randomly selected cells from each of the three independent experiments. Data were shown as the mean \pm standard deviation of three independent experiments $(0.4 \pm$ $0.4 \%$ for untreated vs. $44.0 \pm 1.7 \%$ for 6 -OHDA-treated vs. $0.3 \pm 0.2 \%$ for 3 -MA-treated vs. $12.0 \pm 0.6 \%$ for 6 -OHDA plus 3 -MA-treated cells). ${ }^{* *} P<0.01$; ***P $<0.001$; n.s. not significant

Merker proposed that prenatal tissues undergo three morphologically distinct types of cell death in response to toxicants: type I (apoptotic cell death), type II (autophagic cell death (ACD)), and type III (necrotic cell death) ${ }^{50}$. Apoptosis is a highly regulated process that confers advantages during organism's lifecycle. Indeed, apoptosis is considered as the dominant mechanism of neuronal homeostasis, the dysregulation of which is observed in neurodegenerative diseases including $\mathrm{PD}^{51,52}$. Numerous studies revealed that genetic mutations in PD-related genes led to apoptotic cell death ${ }^{53-55}$. Neurotoxininduced models of PD suggested that apoptosis is primarily responsible for dopaminergic neurodegeneration $^{24,26,27}$. Furthermore, it has been demonstrated that abnormal protein aggregation is commonly observed in various neurodegenerative disorders, and neurons can remove abnormal protein aggregates by two main degradation pathways: ubiquitin-proteasome system and autophagy ${ }^{56,57}$. In particular, autophagy plays an important role in the degradation of long-lived proteins and cellular components in postmitotic neurons ${ }^{58}$. Given the greater dependency of neurons on autophagy for their survival, abnormality in any autophagic flux step can lead to neurodegeneration ${ }^{13,15,16,59}$. In support of this notion, we have demonstrated that impairment of autophagic flux is responsible for dopaminergic neuronal cell death induced by neurotoxins, $\mathrm{MPP}^{+}$, and nigericin ${ }^{30,31}$. In those cases, we found that autophagic degradation process was usually impaired due to lysosomal dysfunction, which caused accumulation of LC3, p62, and ubiquitinated proteins. Intriguingly, that mechanism is in sharp contrast to the findings of the present study where 6-OHDA induced dysregulated autophagy in neurons but the lysosomal activity remained relatively intact. Because
6-OHDA and other neurotoxins with PD-like effects (e.g., $\mathrm{MPP}^{+}$, and rotenone) have been shown to cause cell death by distinct ways ${ }^{22,24,26,27,60}$, we hypothesize that ROS causes dysregulated autophagic induction, whereas intracellular calcium surge could be responsible for autophagic flux impairment (Fig. 8). It would be intriguing to investigate whether and how p62 dynamics are coupled with LC3 dynamics during parkinsonian mimeticsinduced dysregulated autophagy.

ROS are produced as normal products of cellular metabolism and play important roles as signaling molecules regulating various biological phenomena ${ }^{61}$. Because of their highly reactive nature, ROS are directly or indirectly involved in promoting disease progression. In the substantia nigra pars compacta region of PD patients, high levels of ROS are detected, and numerous studies demonstrated that ROS indeed induce apoptosis ${ }^{62}$. Interestingly, recent studies have reported that ROS generation is linked to the induction of autophagic flux ${ }^{63,64}$. In the present study, we investigated the potential role of ROS in the regulation of autophagy using 6-OHDA-treated culture models of PD. We also attempted to reveal whether autophagic signaling contributes to neuronal apoptosis. As was shown by our ultrastructural and biochemical analyses, 6-OHDAinduced neurodegeneration was accompanied by increased autophagic flux, which, in turn depended on ROS generation. The results of our experiment in which 6-OHDA was cotreated with CQ showed that autophagosome accumulation was caused by the dysregulated induction of autophagy rather than by the impairment of autophagic flux. The findings of the study using a tandem mRFP-EGFP-tagged LC3 probe supported this hypothesis. Examination of both signaling molecules and 


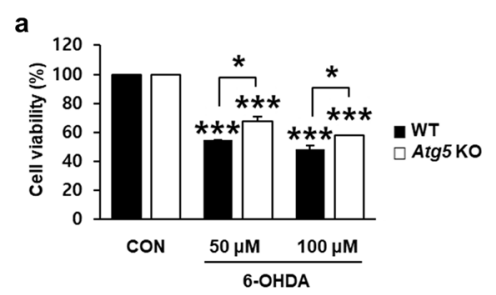

b
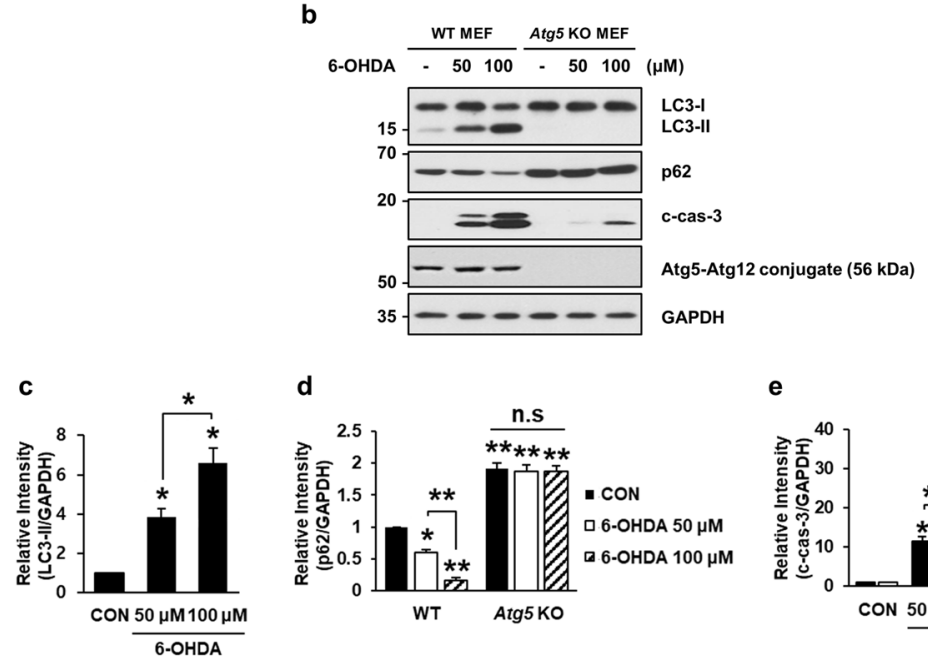

d
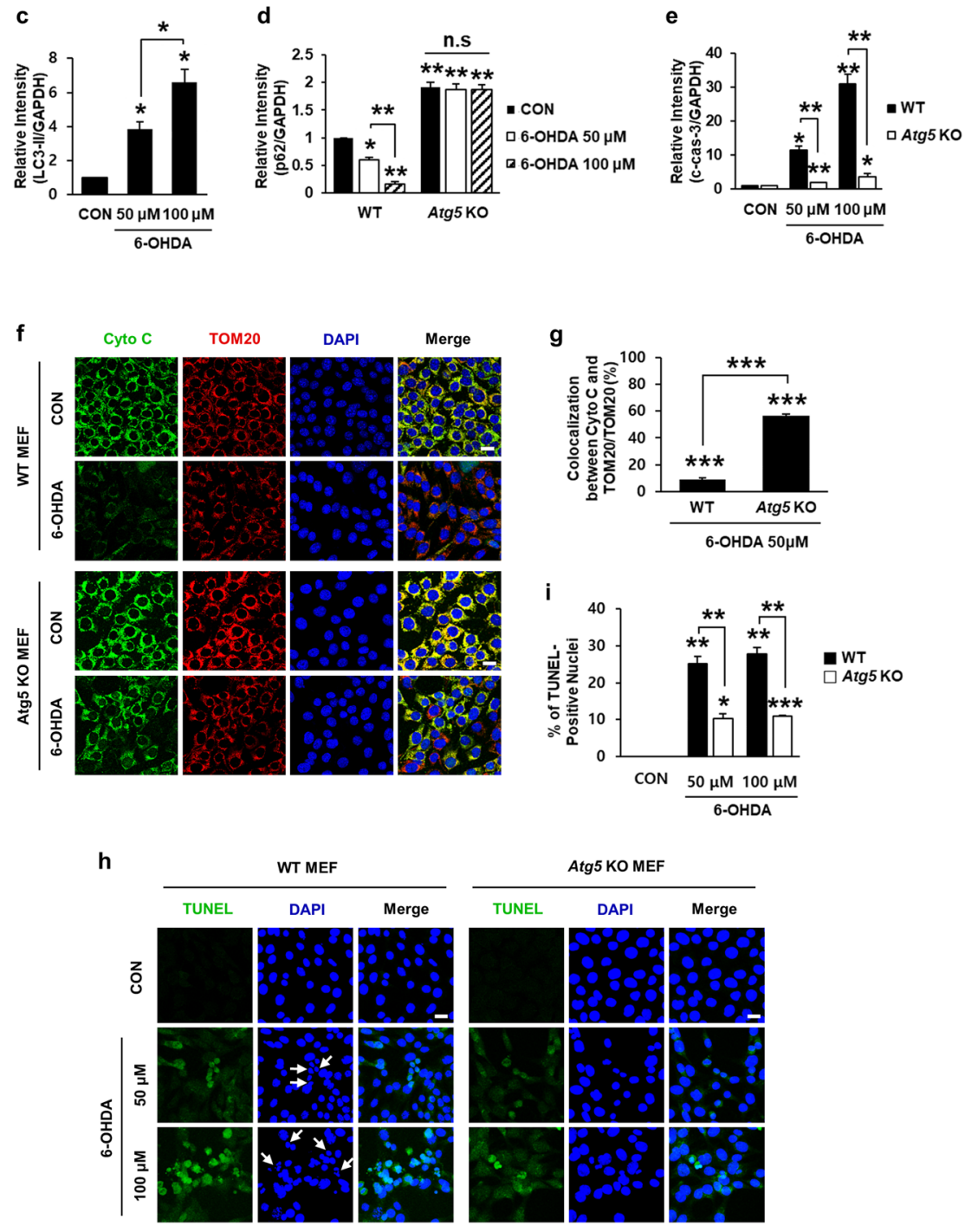

Fig. 6 (See legend on next page.) 
(see figure on previous page)

Fig. 6 Rescue of 6-OHDA-induced neurodegeneration by knockout of Atg5. Wild-type (WT) and Atg5 knockout (KO) MEFs were treated with 50 or $100 \mu \mathrm{M}$ 6-OHDA for $12 \mathrm{~h}$. a MTT reduction assays were performed to assess cell viability. Viability was expressed as percentage over each untreated control cell (100\%). Bars represent the mean \pm standard deviation of three independent experiments (at $50 \mu \mathrm{M}$ 6-OHDA, 54.4 $\pm 0.4 \%$ for WT MEFs vs. $67.9 \pm 2.9 \%$ for Atg $5 \mathrm{KO}$ MEFs; at $100 \mu \mathrm{M}, 6-\mathrm{OHDA} 48.2 \pm 2.5 \%$ for WT MEFs vs. 58.2 $\pm 0.1 \%$ for Atg $5 \mathrm{KO}$ MEFs). ${ }^{*} P<0.05 ;{ }^{* * *} P<0.001$. b Cell lysates were subjected to immunoblot analyses using anti-Atg5, anti-LC3, anti-p62, and anti-c-cas-3. c-e After normalization against GAPDH, densitometric values of LC3-II, p62, and c-cas-3 signals were expressed as a fold change relative to untreated control value. Bars represent the mean \pm standard deviation of three independent experiments (LC3-II, $3.9 \pm 0.4$ for $50 \mu \mathrm{M}$ 6-OHDA-treated vs. $6.6 \pm 0.8$ for $100 \mu \mathrm{M}$ 6-OHDA-treated WT MEFs; p62, $0.6 \pm 0.1$ for $50 \mu \mathrm{M}$ 6-OHDA-treated vs. $0.2 \pm 0.04$ for $100 \mu \mathrm{M}$ 6-OHDA-treated WT MEFs vs. $2.0 \pm 0.1$ for untreated vs. $1.9 \pm 0.1$ for $50 \mu \mathrm{M}$ 6-OHDA-treated vs. $1.9 \pm 0.1$ for $100 \mu \mathrm{M}$ 6-OHDA-treated Atg5 KO MEFs; c-cas-3, $9.8 \pm 1.1$ for $50 \mu \mathrm{M}$ 6-OHDA-treated WT MEFs vs. $1.8 \pm 0.1$ for $50 \mu \mathrm{M}$ 6-OHDA-treated Atg5 KO MEFs; $29.4 \pm 3.0$ for $100 \mu \mathrm{M}$ 6-OHDA-treated WT MEFs vs. $3.7 \pm 0.8$ for $100 \mu \mathrm{M}$ 6-OHDA-treated Atg5 KO MEFs). ${ }^{*} P<0.05$; ${ }^{* *} P<0.01$; n.s. not significant. $\mathbf{f}$ WT and Atg $5 \mathrm{KO}$ MEFs exposed to $50 \mu \mathrm{M}$ 6-OHDA for $12 \mathrm{~h}$ were subjected to immunofluorescent staining for cytochrome $c$ (green), TOM20 (red), and nuclei (blue). Merged images are shown in the right panels. Scale bar represents $20 \mu \mathrm{m}$. $\mathbf{g}$ The percentage of colocalization between cyto $\mathrm{c}$ and TOM20 over total TOM20 was expressed over the untreated control cells (100\%). Confocal images of at least 30 randomly selected cells from each of the three independent experiments were used for quantitation. Bars represent the mean \pm standard deviation of three independent experiments ( $8.9 \pm 0.8 \%$ for WT MEFs vs. $56.5 \pm 0.6 \%$ for Atg5 KO MEFs). ${ }^{* * *} P<0.001$. $\mathbf{h}$ Confocal images of TUNEL assay in WT and Atg5 $\mathrm{KO}$ MEFs exposed to $50 \mu \mathrm{M}$ or $100 \mu \mathrm{M}$ 6-OHDA for $18 \mathrm{~h}$ were examined for quantitation. White arrows indicate condensed or fragmented nuclei. Scale bar represents $20 \mu \mathrm{m}$. i The percentage of TUNEL-positive nuclei was assessed from at least 100 randomly selected cells from each of the three independent experiments. Data represent the mean \pm standard deviation of three independent experiments $(25.2 \pm 2.0 \%$ for $50 \mu \mathrm{M} 6-\mathrm{OHDA}$-treated WT MEFs vs. $10.3 \pm 1.3$ for $50 \mu \mathrm{M}$ 6-OHDA-treated Atg5 KO MEFs; $27.8 \pm 1.6$ for $100 \mu \mathrm{M}$, 6-OHDA-treated WT MEFs vs. 10.9 \pm 0.4 for at $100 \mu \mathrm{M}, 6-\mathrm{OHDA}$ treated Atg5 KO MEFs). ${ }^{*} P<0.05 ;{ }^{* *} P<0.01 ;{ }^{* *} P<0.001$

lysosomal activity also supported our hypothesis that dysregulated autophagy was associated with 6-OHDAinduced neuronal death. Interestingly, we found that 6OHDA-induced autophagy coincided with cytosolic release of cytochrome $c$, caspsase-3 activation, and DNA fragmentation in MN9D cells, MEFs, and primary cultures of cortical neurons. This indicated that autophagic events are somehow involved in 6-OHDA-induced apoptosis. By performing carefully designed experiments using inhibitors of either autophagy or apoptosis, we determined that dysregulated autophagic induction following 6-OHDA treatment was upstream of and linked to caspase-3-dependent apoptosis. Experiments with Atg5 KO MEFs also indicated that autophagy induction preceded caspase-3-dependent apoptosis. Consequently, cotreatment with antioxidant blocked both autophagy induction and subsequent caspase-3 activation, indicating that ROS play a critical role in the regulation of autophagy and autophagy-mediated caspase-dependent neurodegeneration.

The concept of ACD has been initially formulated based on the observations of increased autophagy in dying cells $^{65}$. However, some reports indicate that ACD is not sufficient to promote cell death independent of apoptosis or necrosis ${ }^{66,67}$. Even though the existence of ACD remains controversial, it is largely accepted that interplay between autophagy and apoptotic cell death pathway exists in the pathogenesis. Numerous studies have reported that autophagy precedes apoptosis and regulates apoptotic proteins ${ }^{68,69}$, or apoptosis regulates autophagy $^{70,71}$. These proteins include Bcl-2 family, p53, deathassociated protein kinase, and $\mathrm{JNK}^{44}$. Despite attempts to connect autophagy and apoptosis, their causal relationship wherein one process controls the other has not been adequately demonstrated. In our study, because neuronal death depended on 6-OHDA-induced caspase-3 activation, it is unclear whether dysregulated autophagy itself directly led to ACD. Nevertheless, we explicitly showed that dysregulated autophagy contributed to 6-OHDAtriggered caspase-3-dependent apoptosis. We confirmed this temporal cell death sequence by using pharmacological inhibition and genetic deletion experiments. For example, we demonstrated that cotreatment with 3-MA significantly blocked 6-OHDA-triggered caspase-3 activation and DNA fragmentation and partially protected cells against 6-OHDA toxicity in MN9D cells and mouse cortical neurons. We also found that 6-OHDA-induced caspase-3 activation, DNA fragmentation, and apoptotic neuronal death were attenuated when the induction of autophagy was prevented by the genetic depletion of Atg5. Moreover, we showed that the release of cytochrome $c$ to the cytosol in response to 6-OHDA was significantly decreased in MN9D cells cotreated with 3-MA and in Atg5 KO MEFs. Therefore, our results show a strong causal relationship between autophagy and apoptotic neuronal cell death in which ROS-triggered dysregulated autophagy promotes caspase-dependent apoptotic cell death (Fig. 8).

Further studies delineating the mechanisms underlying the potential role of dysregulated autophagy in apoptotic neurodegeneration are certainly required. First, it would be useful to determine whether autophagic vacuoles are potential sources of $\operatorname{ROS}^{72,73}$. It is possible that increased autophagic structures after the incubation with 6-OHDA set a time bomb for affected cells. Furthermore, it would be of immediate interest to reveal how ROS activates autophagy via recruiting protein kinases and downstream transcriptional factors ${ }^{74,75}$. Secondly, it has been proposed 


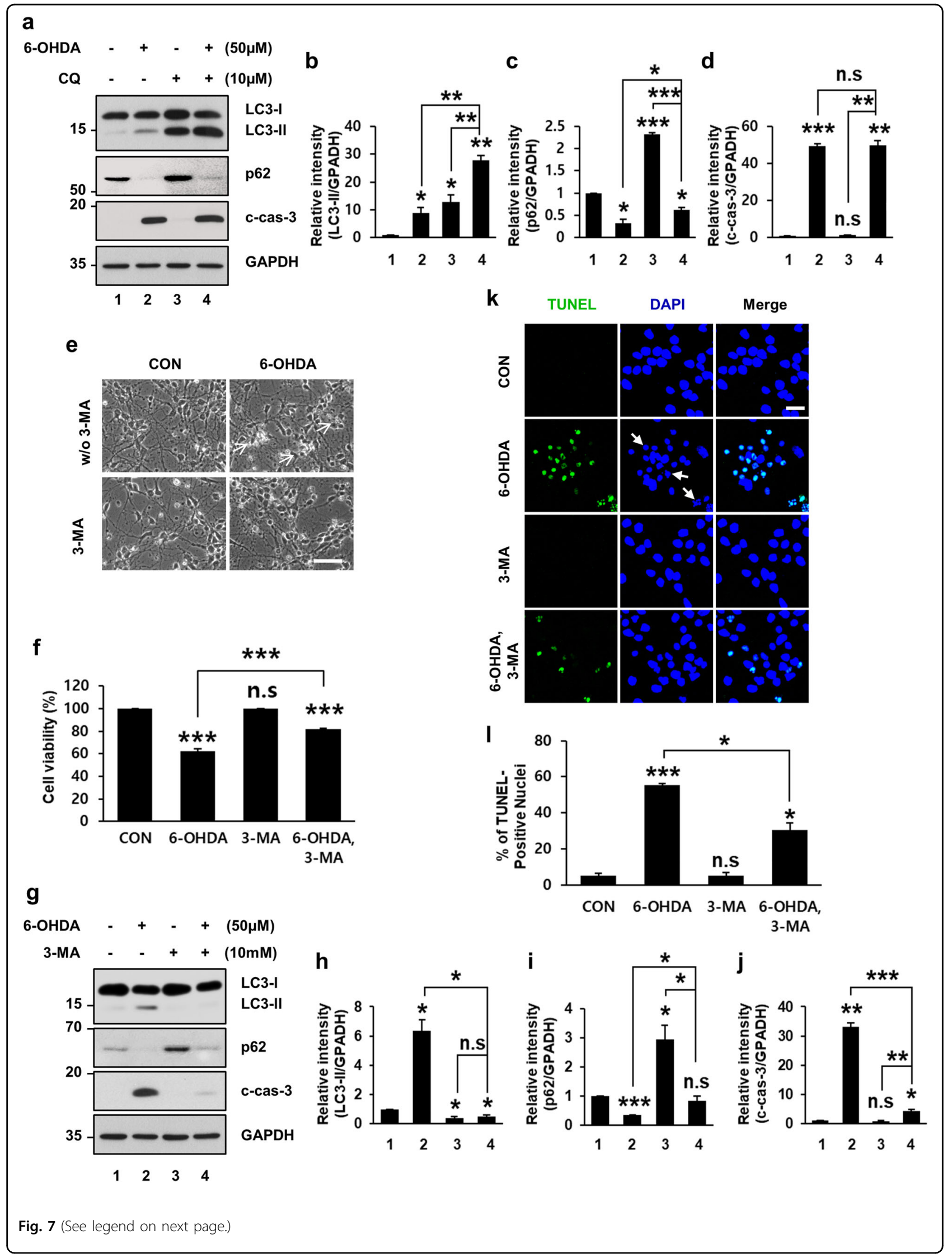


(see figure on previous page)

Fig. 7 Rescue of 6-OHDA-induced cortical neuronal death by 3-MA. Primary cultures of mouse cortical neurons were incubated for $12 \mathrm{~h}$ with 50 $\mu \mathrm{M}$ 6-OHDA in the presence or absence of $10 \mu \mathrm{M}$ CQ (a-d) or $10 \mathrm{mM}$ 3-MA (e-l). a Cell lysates were subjected to immunoblotting using antibodies against LC3, p62, and c-cas-3. b-d After normalization against GAPDH signal intensity, the fold intensity of LC3-II, p62, and c-cas-3 signals were expressed as fold change relative to control value. Data represent the mean \pm standard deviation of three independent experiments (LC3- $\|$, $9.0 \pm 1.8$ for 6-OHDA-treated vs. $12.9 \pm 2.4$ for CQ-treated vs. $27.7 \pm 1.7$ for 6-OHDA plus CQ-treated cells; p62, $0.3 \pm 0.1$ for 6-OHDA-treated vs. $2.3 \pm 0.03$ for CQtreated vs. $0.6 \pm 0.1$ for 6-OHDA plus CQ-treated group; c-cas-3, $49.5 \pm 1.2$ for 6-OHDA-treated vs. $1.2 \pm 0.1$ for CQ-treated vs. $49.6 \pm 2.8$ for 6-OHDA plus CQ-treated group). ${ }^{*} P<0.05$; ${ }^{*} P<0.01$; ${ }^{* *} P<0.001$; n.s. not significant. e Mouse primary cortical neurons were analyzed by a phase-contrast microscopy after drug treatment. Shrunken, phase-bright cells are indicated by white arrows. Scale bar represents $50 \mu \mathrm{m}$. $\mathbf{f}$ Cell viability was measured by the MTT reduction assay. Viability was expressed as percentage over the untreated control cells $(100 \% ; 62.0 \pm 2.2 \%$ for 6 -OHDA-treated vs. $82.1 \pm$ $0.3 \%$ for 6-OHDA plus 3-MA-treated group). Bars represent the mean \pm standard deviation of three independent experiments. ${ }^{* * *} P<0.001$; n.s. not significant. $\mathbf{g}$ The levels of LC3-II, p62, and c-cas-3 were determined by immunoblotting. $\mathbf{h}-\mathbf{j}$ The relative intensities of LC3-II, p62, and c-cas-3 signals were calculated after normalization against GAPDH signal intensity. Bars represent the mean \pm standard deviation of three independent experiments (LC3-II, $6.3 \pm 0.8$ for 6-OHDA-treated vs. $0.4 \pm 0.1$ for 3-MA-treated vs. $0.5 \pm 0.1$ for 6-OHDA plus 3-MA-treated group; p62, 0.3 \pm 0.001 for 6-OHDAtreated vs. $3.0 \pm 0.5$ for 3-MA-treated vs. $0.9 \pm 0.1$ for 6-OHDA plus 3-MA-treated group; for c-cas-3, $33.2 \pm 1.3$ for 6-OHDA-treated vs. 0.9 \pm 0.2 for 3-MAtreated vs. $4.4 \pm 0.5$ for $6-\mathrm{OHDA}$ plus 3 -MA-treated group). ${ }^{*} P<0.05$; ${ }^{* *} P<0.01$; ${ }^{* * *} P<0.001$; n.s. not significant. $\mathbf{k}$ TUNEL assay was performed in primary cultures of mouse cortical neurons exposed to $50 \mu \mathrm{M} 6-\mathrm{OHDA}$ for $18 \mathrm{~h}$ in the presence or absence of $10 \mathrm{mM}$ 3-MA. White arrows indicate condensed or fragmented nuclei. Scale bar represents $20 \mu \mathrm{m}$. I The percentage of TUNEL-positive nuclei was assessed from at least 100 randomly selected cells from each of the three independent experiments. Data represent the mean \pm standard deviation of three independent experiments $(5.4 \pm 1.3 \%$ for untreated vs. $55.6 \pm 0.8 \%$ for 6-OHDA-treated vs. $5.2 \pm 1.9 \%$ for 3-MA-treated vs. $30.5 \pm 3.8 \%$ for 6-OHDA plus 3-MA-treated group). ${ }^{*} P<0.05 ;{ }^{* * *} P<0.001$, n.s. not significant

that many neurodegenerative diseases may result from dysregulated autophagy, particularly from the impairment of lysosomal degradative mechanisms ${ }^{76,77}$. Experimental evidence suggests that dysregulated autophagy puts a significant pressure on compromised lysosomes through increasing their cargos that needs to be degraded $^{73}$. Therefore, it should be determined whether inhibition of dysregulated autophagy indeed provides benefit to cells by reducing the risk of lysosomal membrane destabilization $^{78}$. Carefully designed experiments in these areas will expand our understanding of the role of autophagy in neurodegenerative disorders.

\section{Materials and methods Chemicals}

The chemicals used for this study included 6-OHDA (Regis Chemical, Chicago, USA), chloroquine diphosphate salt (CQ; Sigma-Aldrich, St. Louis, MO, USA), NAC (Sigma-Aldrich), Z-VAD-FMK (Enzo Life Science Inc., Farmingdale, NY, USA), and 3-MA (Sigma-Aldrich). The concentrations and incubation periods of all drugs used in this study were empirically determined and used as described previously ${ }^{22,24,26,27,30,31}$.

\section{Cell culture and drug treatment}

MN9D dopaminergic neuronal cell line was established by somatic fusion between embryonic mesencephalic neurons and N18TG neuroblastoma ${ }^{28,29}$ and cultured as previously described ${ }^{30,31}$. Briefly, MN9D cells were cultivated at $37^{\circ} \mathrm{C}$ in Dulbecco's modified Eagle's medium (DMEM; Sigma-Aldrich) supplemented with $10 \%$ heatinactivated fetal bovine serum (FBS; Gibco, Grand Island, $\mathrm{NY}$, USA) on dishes coated with $25 \mu \mathrm{g} / \mathrm{mL}$ poly-D-lysine
(Sigma-Aldrich) in the atmosphere of $90 \%$ air and $10 \%$ $\mathrm{CO}_{2}$. For WT and Atg5 KO MEFs, the culture medium containing DMEM (GenDEPOT, Barker, TX, USA) supplemented with $10 \%$ FBS (GenDEPOT) was used, and the cells were incubated at $37^{\circ} \mathrm{C}$ in the atmosphere of $95 \%$ air and $5 \% \mathrm{CO}_{2}$. For drug treatment, culture medium was changed to $\mathrm{N} 2$ serum-free defined medium containing the indicated drugs and further incubated for the time periods indicated.

\section{Primary cultures of cortical neurons}

All mice were handled in accordance with the guideline for animal care and use of the Yonsei University. All experimental procedures were approved by the Institutional Animal Care and Use Committee of the Yonsei University (permissions IACUC (2017-10-647-01 and 2018-01-689-01)). Cerebral cortices were removed from gestational day 14.5 mouse embryos (Orient, Gyeong-gi, Republic of Korea) and mechanically dissociated as previously described ${ }^{79}$. Briefly, dissociated cortical cells were plated at a density of $5 \times 10^{6}$ cells per well of six-well plates or at $1 \times 10^{6}$ cells per well of 24 -well plates that were precoated with $100 \mu \mathrm{g} / \mathrm{mL}$ poly-D-lysine and $1 \mu \mathrm{g} / \mathrm{mL}$ laminin (Invitrogen, Carlsbad, CA, USA). Cultures were incubated at $37^{\circ} \mathrm{C}$ in Minimum Essential Medium (MEM, Gibco) supplemented with $0.6 \%$ glucose (SigmaAldrich), $1 \mathrm{mM}$ sodium pyruvate (Sigma-Aldrich), $2 \mathrm{mM}$ L-glutamine (Sigma-Aldrich), penicillin-streptomycin $(100 \mathrm{U} / \mathrm{mL}$ ), and $10 \%$ FBS (Gibco) in the atmosphere of $95 \%$ air and $5 \% \mathrm{CO}_{2}$. After $24 \mathrm{~h}$, culture medium was changed to Neurobasal medium (Invitrogen) supplemented with $2 \%$ B-27 (Gibco), $0.5 \mathrm{mM}$ L-glutamine and $10 \mu \mathrm{M}$ cytosine $\beta$-D-arabinofuranoside (Ara-C, 


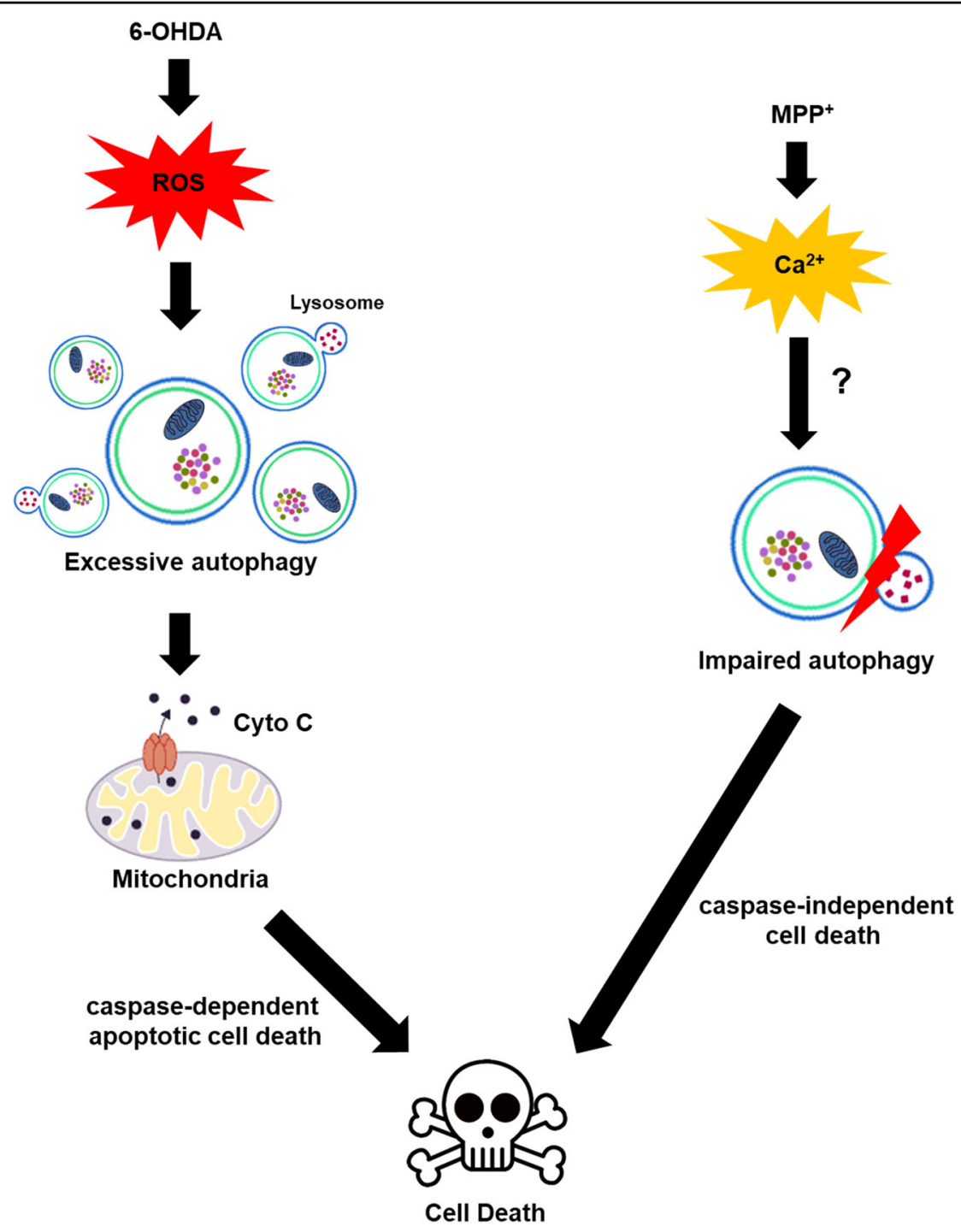

Fig. 8 Schematic diagram of 6-OHDA-induced neurodegeneration. Previously, we demonstrated that 6-OHDA-induced generation of ROS triggered caspase-dependent neuronal death whereas MPP ${ }^{+}$-induced intracellular calcium surge caused caspase-independent cell death ${ }^{22,24,26,27,60}$. Based on the data obtained from pharmacological inhibition (3-MA, CQ) and genetic deletion (Atg5) experiments, in this study, we further propose that potentially damaging levels of ROS induced by 6-OHDA treatment trigger dysregulated autophagic induction and this upstream event, in turn, causes the release of cytochrome $c$ from the mitochondria to the cytosol, eventually accelerating caspase-3-dependent apoptosis. This finding is in sharp contrast to our previous finding that $\mathrm{MPP}^{+}$impaired autophagic flux by inhibiting lysosomal activity ${ }^{30,} 31$

Sigma-Aldrich). At 4 DIV, cultures were treated with the indicated drugs that were dissolved in the same medium.

\section{Electron microscopy}

For electron microscopy, MN9D cells grown in petri dishes were fixed with a mixture of $2 \%$ formaldehyde (freshly prepared from paraformaldehyde) and $0.2 \%$ glutaraldehyde in $0.1 \mathrm{M}$ cacodylate buffer ( $\mathrm{pH} 7.2)$ for $30 \mathrm{~min}$ at $37^{\circ} \mathrm{C}$. To stop fixation, free aldehyde groups were blocked by soaking the cells in $50 \mathrm{mM} \mathrm{NH}_{4} \mathrm{Cl}$ in cacodylate buffer for $1 \mathrm{~h}$. After rinses with the buffer, the cells were mechanically removed, sedimented by centrifugation, enclosed in liquified $2 \%$ agarose, and postfixed with $1 \%$ osmium tetroxide (Electron Microscopy Science (EMS); Hatfield, PA, USA) in distilled water for $1 \mathrm{~h}$, followed by en bloc staining for $1 \mathrm{~h}$ with $1 \%$ aqueous uranyl acetate (Heraeus, Hanau, Germany). After preparations were dehydrated in a series of graded ethanol, embedding in Epon-Araldite (Fluka, Buchs, Germany) was performed according to standard protocol. To unambiguously identify the autophagic structures, ten serial ultrathin sections $(80 \mathrm{~nm})$ were prepared on copper slot grids and stained with uranyl acetate and lead citrate and observed at $80 \mathrm{kV}$ with a Hitachi H-7650 electron 
microscope (Hitachi, Tokyo, Japan). Ten randomly selected cells per each group were used for quantitation of autophagic vacuoles. Electron micrographs were taken with an 11 megapixel CCD XR611-M digital camera (Advanced Microscopy Techniques; Woburn, MA, USA).

\section{Phase-contrast and confocal microscopy}

To observe morphological changes in MN9D cells and primary cultures of cortical neurons following treatment with the indicated drugs, the cells were photographed under an Axio Observer A1 microscope (Carl Zeiss, Zena, Germany). For immunocytochemical analysis, MN9D cells and MEFs were cultured on cover glasses coated with $100 \mu \mathrm{g} / \mathrm{mL}$ poly-D-lysine (Sigma-Aldrich). At time periods indicated, the cells were fixed in $4 \%$ paraformaldehyde (EMS) in PBS for $10 \mathrm{~min}$ at room temperature (RT). The permeabilization step was carried out with $0.1 \%$ saponin (Sigma-Aldrich) for $10 \mathrm{~min}$ at RT. Subsequently, the cells were blocked in PBS containing 0.2\% Triton X-100 and $5 \%$ normal goat serum (Invitrogen), and then incubated with primary antibodies. These included a rabbit anti-LC3 antibody (Cell Signaling, Beverly, MA, USA; 1:200), a mouse anti-cytochrome $c$ antibody (BD Biosciences, Bedford, MA, USA; 1:200), and a rabbit anti-TOM20 antibody (Santa Cruz Biotechnology, Inc., CA, USA; 1:200), in PBS containing 0.2\% Triton X-100 and 1\% normal goat serum at $4{ }^{\circ} \mathrm{C}$ for overnight. After extensive washes with PBS, the cells were incubated with appropriate secondary antibodies for $1 \mathrm{~h}$ at RT. These included Alexa 488-conjugated goat anti-rabbit IgG, Alexa 488conjugated goat anti-mouse IgG, and Alexa 546conjugated goat anti-rabbit IgG (all from Invitrogen, 1:200). Hoechst 33258 (Molecular Probes, Inc., Eugene, Oregon, USA; $1 \mu \mathrm{g} / \mathrm{mL}$ ) was used for nuclei staining. After extensive washes with PBS, slides were mounted with Vectashield mounting medium (Vector Laboratories, Burlingame, CA, USA). Z stacked series of fluorescence images were acquired under a confocal microscope equipped with epifluorescence and digital image analyzer (LSM 700, Carl Zeiss). To quantify the LC3 punctate staining pattern per cell, at least 30 cells were randomly selected from each of three independent experiments and analyzed using ImageJ Imaging software (National Institutes of Health, Bethesda, MD, USA) as previously described $^{30}$. Dots with diameters of $0.2-10 \mu \mathrm{m}$ were included in our count. To assess the percentage of colocalization with cytochrome $c$ and TOM20, at least 30 cells were randomly selected, and the areas of colocalized cytochrome $c$ and TOM20 signal were divided by the total area of TOM20 signal using ImageJ software. MN9D cells treated with or without 6-OHDA were loaded with $0.5 \mu \mathrm{M}$ LysoTracker Red DND-99 (LTR, Invitrogen), a fluorescent probe highly selective for acidic organelles, to conduct confocal imaging of lysosomal activity. The intensity of the LTR signals was quantified from at least 100 randomly selected cells from each of three independent experiments using ImageJ software. To explore the ectopic expression of a tandem mRFP-EGFP-LC3 probe, MN9D cells were transiently transfected using Lipofectamine $^{\mathrm{TM}} 2000$ transfection reagent (Invitrogen). Following drug treatment, at least 30 randomly selected cells from each of three independent experiments were subjected to quantitative analyses for the number of puncta that were either $\mathrm{EGFP}^{-} / \mathrm{mRFP}^{+}$or $\mathrm{EGFP}^{+} / \mathrm{mRFP}^{+}$.

\section{Immunoblot analysis}

After drug treatment, the cells were washed with cold PBS (Lonza, Basel, Switzerland) with $2 \mathrm{mM}$ ethylenediaminetetraacetic acid (EDTA; Sigma-Aldrich), lysed on ice in RIPA buffer (50 mM Tris-HCl [pH 7.4], $1 \%$ NP-40, $0.25 \%$ sodium deoxycholate, $150 \mathrm{mM} \mathrm{NaCl}, 1 \mathrm{mM}$ EDTA, $0.1 \%$ sodium dodecyl sulfate (SDS)) containing complete protease inhibitor cocktail (Roche Applied Science, Mannheim, Germany), and then homogenized using a 1 - $\mathrm{mL}$ syringe with a 20 -gauge needle. Lysates were centrifuged at $13,000 \times g$ for $20 \mathrm{~min}$ at $4{ }^{\circ} \mathrm{C}$. Total supernatant protein was collected and quantified using Bradford protein assay reagent (Bio-Rad, Hercules, CA, USA). Predetermined amounts of protein from each preparation were separated on a sodium dodecyl sulfate polyacrylamide gel, transferred onto polyvinylidene fluoride membranes (Pall Corp., Ann Arbor, MI, USA), and blocked with Tris-buffered saline containing $0.1 \%$ Tween20 (TBST) and 5\% skim milk over $30 \mathrm{~min}$. The membranes were incubated with primary antibodies overnight at $4{ }^{\circ} \mathrm{C}$. Unless otherwise specified, all primary antibodies used were obtained from Cell Signaling. Primary antibodies used included a rabbit anti-LC3 antibody (1:4000), a rabbit anti-cleaved caspase-3 antibody (1:1000), a rabbit anti-p-mTOR antibody (1:1000), a rabbit anti-mTOR antibody (1:1000), a rabbit anti-p-p70S6K antibody (1:1000), a rabbit anti-p70S6K antibody (1:1000), a rabbit anti-p-AMPK antibody (1:1000), a rabbit anti-AMPK antibody (1:1000), a rabbit anti-p-Akt antibody (1:1000), a rabbit anti-Akt antibody (1:1000), a rabbit anti-Rab5 antibody (Cell Signaling, 1:1000), a rabbit anti-Rab7 antibody (Cell Signaling, 1:1000), a mouse anti-cathepsin D antibody (Santa Cruz; 1:1000), a guinea pig anti-p62/ SQSTM1 (Progen, Heidelberg, Germany; 1:5000), a rabbit anti-Atg5 antibody (Novus Biologicals, Littleton, CO, USA; $1: 1000$ ) and anti-FLAG M2-Peroxidase (HRP) antibody (Sigma-Aldrich; 1:3000). Mouse anti-GAPDH (Millipore, Billerica, MA, 1:10,000) was used as loading control. After extensive washes with TBST, blots were incubated with horseradish peroxidase-conjugated antirabbit (Santa Cruz; 1:5000) or anti-mouse (Santa Cruz; 1:5000) secondary antibody. Specific bands were detected by using an enhanced chemiluminescence kit (ECL; 
PerkinElmer, Waltham, MA, USA). The relative band intensity was measured using ImageJ Imaging Software and expressed as a value normalized by the intensity of GAPDH signal.

\section{Viability assays}

To examine cell viability after indicated drug treatments, MTT reduction assay and TUNEL assay were performed. For MTT reduction assay ${ }^{80}$, cells cultured on 24-well plates were incubated with $1 \mathrm{mg} / \mathrm{mL}$ MTT solution (Sigma-Aldrich) at $37^{\circ} \mathrm{C}$ for $1 \mathrm{~h}$ and lysed for $18 \mathrm{~h}$ in an extraction buffer containing 20\% SDS in 50\% aqueous dimethylformamide. The optical densities of formazan grain were measured at 590 and $650 \mathrm{~nm}$ as test and reference wavelengths, respectively, by a $\operatorname{VICTOR}^{\text {in }}$ X5 Multilabel Plate Reader (PerkinElmer). Cell viability was expressed as percentage relative to the value in untreated control (100\%). For TUNEL assays, cells were prepared using the same methods described previously for immunocytochemistry and confocal microscopy. After the permeabilization step, the cells were incubated with the In Situ Cell Death Detection Kit, Fluorescein (Roche) at $37^{\circ} \mathrm{C}$ for $1 \mathrm{~h}$. After extensive washes with PBS, the cells were incubated with PBS containing $1 \mu \mathrm{g} / \mathrm{mL}$ Hoechst 33258 (Molecular Probes, Inc.) at RT for $5 \mathrm{~min}$, and then the slides were mounted with Vectashield mounting medium (Vector Laboratories). Z stacked series of fluorescence images were examined under a confocal microscope equipped with epifluorescence and digital image analyzer (LSM 700, Carl Zeiss). The percentage of TUNEL-positive nuclei was assessed from at least 100 randomly selected cells from each of three independent experiments.

\section{Statistics}

Data are expressed as the mean \pm standard deviation of three independent experiments. To determine the significance of differences between groups, the two-tailed Student's $t$-tests or one-way analysis of variance followed by the Tukey's post hoc test were performed by using Prism 6 (GraphPad Inc, San Diego, CA, USA). Statistical significance of differences was indicated as follows: *** $P<0.001 ;{ }^{* * *} P<0.01$; or " $P<0.05$.

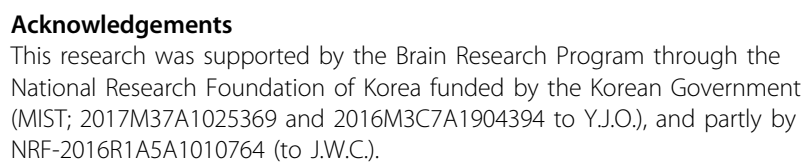
National Research Foundation of Korea funded by the Korean Government (MIST; 2017 M37A1025369 and 2016M3C7A1904394 to Y.J.O.), and partly by NRF-2016R1A5A1010764 (to J.W.C.).

\section{Author details}

${ }^{1}$ Department of Systems Biology, Yonsei University College of Life Science and Biotechnology, Seoul 120-749, South Korea. ${ }^{2}$ Glycosylation Network Research Center, Yonsei University, Seoul 120-749, South Korea. ${ }^{3}$ Interdisciplinary Program of Integrated OMICS for Biomedical Science, Yonsei University, Seoul 120-749, South Korea
Conflict of interest

The authors declare that they have no conflict of interest.

\section{Publisher's note}

Springer Nature remains neutral with regard to jurisdictional claims in published maps and institutional affiliations.

Supplementary Information accompanies this paper at (https://doi.org/ 10.1038/s41419-018-1229-y).

Received: 23 March 2018 Revised: 29 October 2018 Accepted: 20 November 2018

Published online: 11 December 2018

\section{References}

1. Levine, B. \& Kroemer, G. Autophagy in the pathogenesis of disease. Cell 132, $27-42$ (2008).

2. Alirezaei, M. et al. Short-term fasting induces profound neuronal autophagy. Autophagy 6, 702-710 (2010).

3. Eisenberg, $T$. et al. Induction of autophagy by spermidine promotes longevity. Nat. Cell Biol. 11, 1305-1314 (2009).

4. Levine, B. Cell biology: autophagy and cancer. Nature 446, 745-747 (2007).

5. Puyal, J., Ginet, V., Grishchuk, Y., Truttmann, A. C. \& Clarke, P. G. Neuronal autophagy as a mediator of life and death: contrasting roles in chronic neurodegenerative and acute neural disorders. Neuroscientist 18, 224-236 (2012).

6. Scarlatti, F., Granata, R., Meijer, A. J. \& Codogno, P. Does autophagy have a license to kill mammalian cells? Cell Death Differ. 16, 12-20 (2009).

7. Carra, S., Seguin, S. J., Lambert, H. \& Landry, J. HspB8 chaperone activity toward poly(Q)-containing proteins depends on its association with Bag3, a stimulator of macroautophagy. J. Biol. Chem. 283, 1437-1444 (2008).

8. Cuervo, A. M., Stefanis, L., Fredenburg, R., Lansbury, P. T. \& Sulzer, D. Impaired degradation of mutant alpha-synuclein by chaperone-mediated autophagy. Science 305, 1292-1295 (2004).

9. Lipinski, M. M. et al. Genome-wide analysis reveals mechanisms modulating autophagy in normal brain aging and in Alzheimer's disease. Proc. Natl Acad. Sci. USA 107, 14164-14169 (2010).

10. Wang, I. F. et al. Autophagy activators rescue and alleviate pathogenesis of a mouse model with proteinopathies of the TAR DNA-binding protein 43. Proc. Natl Acad. Sci. USA 109, 15024-15029 (2012).

11. Jiang, T. et al. Temsirolimus promotes autophagic clearance of amyloid-beta and provides protective effects in cellular and animal models of Alzheimer's disease. Pharmacol. Res. 81, 54-63 (2014).

12. Vidoni, C., Secomandi, E., Castiglioni, A., Melone, M. A. B. \& Isidoro, C. Resveratrol protects neuronal-like cells expressing mutant Huntingtin from dopamine toxicity by rescuing ATG4-mediated autophagosome formation. Neurochem. Int. 117, 174-187 (2017).

13. He, X., Yuan, W., Li, Z. \& Feng, J. An autophagic mechanism is involved in the 6-hydroxydopamine-induced neurotoxicity in vivo. Toxicol. Lett. 280, 29-40 (2017).

14. Lee, J. A. \& Gao, F. B. Inhibition of autophagy induction delays neuronal cell loss caused by dysfunctional ESCRT-III in frontotemporal dementia. J. Neurosci. 29, 8506-8511 (2009).

15. Ginet, $\mathrm{V}$. et al. Involvement of autophagy in hypoxic-excitotoxic neuronal death. Autophagy 10, 846-860 (2014).

16. Grishchuk, Y., Ginet, V., Truttmann, A. C., Clarke, P. G. \& Puyal, J. Beclin $1-$ independent autophagy contributes to apoptosis in cortical neurons. Autophagy 7, 1115-1131 (2011).

17. Wirawan, E. et al. Caspase-mediated cleavage of Beclin-1 inactivates Beclin-1-induced autophagy and enhances apoptosis by promoting the release of proapoptotic factors from mitochondria. Cell Death Dis. 1, e18 (2010).

18. Dauer, W. \& Przedborski, S. Parkinson's disease: mechanisms and models. Neuron 39, 889-909 (2003).

19. Ungerstedt, U. 6-Hydroxy-dopamine induced degeneration of central monoamine neurons. Eur. J. Pharmacol. 5, 107-110 (1968). 
20. Bove, J. et al. Neuroprotection induced by the adenosine A2A antagonist CSC in the 6-OHDA rat model of parkinsonism: effect on the activity of striatal output pathways. Exp. Brain Res. 165, 362-374 (2005).

21. Glinka, Y., Gassen, M. \& Youdim, M. B. Mechanism of 6-hydroxydopamine neurotoxicity. J. Neural Transm. Suppl. 50, 55-66 (1997).

22. Choi, W. S. et al. Two distinct mechanisms are involved in 6-hydroxydopamine- and MPP+-induced dopaminergic neuronal cell death: role of caspases, ROS, and JNK. J. Neurosci. Res. 57, 86-94 (1999).

23. Gomez-Lazaro, M. et al. 6-Hydroxydopamine activates the mitochondrial apoptosis pathway through p38 MAPK-mediated, p53-independent activation of Bax and PUMA. J. Neurochem. 104, 1599-1612 (2008).

24. Han, B. S., Noh, J. S., Gwag, B. J. \& Oh, Y. J. A distinct death mechanism is induced by 1-methyl-4-phenylpyridinium or by 6-hydroxydopamine in cultured rat cortical neurons: degradation and dephosphorylation of tau. Neurosci. Lett. 341, 99-102 (2003).

25. Solesio, M. E., Saez-Atienzar, S., Jordan, J. \& Galindo, M. F. Characterization of mitophagy in the 6-hydoxydopamine Parkinson's disease model. Toxicol. Sci. 129, 411-420 (2012)

26. Han, B. S. et al. Caspase-dependent and -independent cell death pathways in primary cultures of mesencephalic dopaminergic neurons after neurotoxin treatment. J. Neurosci. 23, 5069-5078 (2003).

27. Lee, Y. M. et al. Oxidative modification of peroxiredoxin is associated with drug-induced apoptotic signaling in experimental models of Parkinson disease. J. Biol. Chem. 283, 9986-9998 (2008).

28. Choi, H. K., Won, L., Roback, J. D., Wainer, B. H. \& Heller, A. Specific modulation of dopamine expression in neuronal hybrid cells by primary cells from different brain regions. Proc. Natl Acad. Sci. USA 89, 8943-8947 (1992).

29. Choi, H. K. et al. Immortalization of embryonic mesencephalic dopaminergic neurons by somatic cell fusion. Brain Res. 552, 67-76 (1991).

30. Lim, J. et al. Binding preference of p62 towards LC3-II during dopaminergic neurotoxin-induced impairment of autophagic flux. Autophagy 7, 51-60 (2011).

31. Lim, J. et al. Nigericin-induced impairment of autophagic flux in neuronal cells is inhibited by overexpression of Bak. J. Biol. Chem. 287, 23271-23282 (2012).

32. Klionsky, D. J. et al. Guidelines for the use and interpretation of assays for monitoring autophagy (3rd edition). Autophagy 12, 1-222 (2016).

33. Mizushima, N., Yoshimori, T. \& Levine, B. Methods in mammalian autophagy research. Cell 140, 313-326 (2010).

34. Yla-Anttila, P., Vihinen, H., Jokitalo, E. \& Eskelinen, E. L. Monitoring autophagy by electron microscopy in Mammalian cells. Methods Enzymol. 452, 143-164 (2009).

35. Kabeya, Y. et al. LC3, a mammalian homologue of yeast Apg8p, is localized in autophagosome membranes after processing. EMBO J. 19, 5720-5728 (2000)

36. Rubinsztein, D. C. et al. In search of an "autophagomometer". Autophagy $\mathbf{5}$, 585-589 (2009)

37. Kim, S. J., Park, Y. J. \& Oh, Y. J. Proteomic analysis reveals a protective role for DJ-1 during 6-hydroxydopamine-induced cell death. Biochem. Biophys. Res. Commun. 422, 8-14 (2012).

38. Park, S. H., Choi, W. S., Yoon, S. Y., Ahn, Y. S. \& Oh, Y. J. Activation of NF-kappaB is involved in 6-hydroxydopamine-but not MPP+-induced dopaminergic neuronal cell death: its potential role as a survival determinant. Biochem. Biophys. Res. Commun. 322, 727-733 (2004).

39. Ebadi, M., Hiramatsu, M., Burke, W. J., Folks, D. G. \& el-Sayed, M. A. Metallothionein isoforms provide neuroprotection against 6-hydroxydopaminegenerated hydroxyl radicals and superoxide anions. Proc. West. Pharmacol. Soc. 41, 155-158 (1998).

40. Wang, X., Chen, S., Ma, G., Ye, M. \& Lu, G. Involvement of proinflammatory factors, apoptosis, caspase-3 activation and Ca2+disturbance in microglia activation-mediated dopaminergic cell degeneration. Mech. Ageing Dev. 126, 1241-1254 (2005).

41. Zhang, X. J., Chen, S., Huang, K. X. \& Le, W. D. Why should autophagic flux be assessed? Acta Pharmacol. Sin. 34, 595-599 (2013).

42. Ju, J. S., Varadhachary, A. S., Miller, S. E. \& Weihl, C. C. Quantitation of "autophagic flux" in mature skeletal muscle. Autophagy 6, 929-935 (2010).

43. Matsuzawa, Y. et al. RIPK3 regulates p62-LC3 complex formation via the caspase-8-dependent cleavage of p62. Biochem. Biophys. Res. Commun. 456, 298-304 (2015).

44. Yang, Z. \& Klionsky, D. J. Mammalian autophagy: core molecular machinery and signaling regulation. Curr. Opin. Cell Biol. 22, 124-131 (2010).
45. Blommaart, E. F., Krause, U., Schellens, J. P., Vreeling-Sindelarova, H. \& Meijer, A. J. The phosphatidylinositol 3-kinase inhibitors wortmannin and LY294002 inhibit autophagy in isolated rat hepatocytes. Eur. J. Biochem. 243, 240-246 (1997).

46. Liu, X., Kim, C. N., Yang, J., Jemmerson, R. \& Wang, X. Induction of apoptotic program in cell-free extracts: requirement for dATP and cytochrome c. Cell $\mathbf{8 6}$, 147-157 (1996).

47. Kroemer, G., Dallaporta, B. \& Resche-Rigon, M. The mitochondrial death/life regulator in apoptosis and necrosis. Annu. Rev. Physiol. 60 619-642 (1998).

48. Loo, J. F., Lau, P. M., Ho, H. P. \& Kong, S. K. An aptamer-based bio-barcode assay with isothermal recombinase polymerase amplification for cytochrome-c detection and anti-cancer drug screening. Talanta $\mathbf{1 1 5}$ 159-165 (2013)

49. He, X. et al. 6-Hydroxydopamine induces autophagic flux dysfunction by impairing transcription factor EB activation and lysosomal function in dopaminergic neurons and SH-SY5Y cells. Toxicol. Lett. 283, 58-68 (2018).

50. Schweichel, J. U. \& Merker, H. J. The morphology of various types of cell death in prenatal tissues. Teratology 7, 253-266 (1973).

51. Agostini, M., Tucci, P. \& Melino, G. Cell death pathology: perspective for human diseases. Biochem. Biophys. Res. Commun. 414, 451-455 (2011).

52. Kapetanakis, N. et al. Helicobacter pylori infection and colorectal carcinoma: pathologic aspects. J. Gastrointest. Oncol. 3, 377-379 (2012).

53. Ho, C. C., Rideout, H. J., Ribe, E., Troy, C. M. \& Dauer, W. T. The Parkinson disease protein leucine-rich repeat kinase 2 transduces death signals via Fasassociated protein with death domain and caspase-8 in a cellular model of neurodegeneration. J. Neurosci. 29, 1011-1016 (2009).

54. Jin, $H$. et al. alpha-Synuclein negatively regulates protein kinase Cdelta expression to suppress apoptosis in dopaminergic neurons by reducing p300 histone acetyltransferase activity. J. Neurosci. 31, 2035-2051 (2011).

55. Yamamoto, A. et al. Parkin phosphorylation and modulation of its E3 ubiquitin ligase activity. J. Biol. Chem. 280, 3390-3399 (2005).

56. Xilouri, M. \& Stefanis, L. Autophagy in the central nervous system: implications for neurodegenerative disorders. Cns. Neurol. Disord. Drug Targets 9, 701-719 (2010).

57. Nijholt, D. A., De Kimpe, L., Elfrink, H. L., Hoozemans, J. J. \& Scheper, W. Removing protein aggregates: the role of proteolysis in neurodegeneration Curr. Med. Chem. 18, 2459-2476 (2011).

58. Tooze, S. A. \& Schiavo, G. Liaisons dangereuses: autophagy, neuronal survival and neurodegeneration. Curr. Opin. Neurobiol. 18, 504-515 (2008).

59. Cheng, H. C. et al. Akt suppresses retrograde degeneration of dopaminergic axons by inhibition of macroautophagy. J. Neurosci. 31, 2125-2135 (2011).

60. Choi, W. S. et al. Phosphorylation of p38 MAPK induced by oxidative stress is linked to activation of both caspase-8- and -9-mediated apoptotic pathways in dopaminergic neurons. J. Biol. Chem. 279, 20451-20460 (2004).

61. Zuo, L., Zhou, T., Pannell, B. K., Ziegler, A. C. \& Best, T. M. Biological and physiological role of reactive oxygen species-the good, the bad and the ugly. Acta Physiol. 214, 329-348 (2015)

62. Dias, V., Junn, E. \& Mouradian, M. M. The role of oxidative stress in Parkinson's disease. J. Park. Dis. 3, 461-491 (2013).

63. Jangamreddy, J. R. et al. Salinomycin induces activation of autophagy, mitophagy and affects mitochondrial polarity: differences between primary and cancer cells. Biochim. Biophys. Acta 1833, 2057-2069 (2013).

64. Kaminskyy, V. O. \& Zhivotovsky, B. Free radicals in cross talk between autophagy and apoptosis. Antioxid. Redox Signal. 21, 86-102 (2014).

65. Shen, H. M. \& Codogno, P. Autophagic cell death: Loch Ness monster or endangered species? Autophagy 7, 457-465 (2011).

66. Nixon, R. A. \& Yang, D. S. Autophagy and neuronal cell death in neurological disorders. Cold Spring Harb. Perspect. Biol. 4, 4 (2012).

67. Shen, S. et al. Association and dissociation of autophagy, apoptosis and necrosis by systematic chemical study. Oncogene 30, 4544-4556 (2011).

68. Chang, N. C., Nguyen, M., Germain, M. \& Shore, G. C. Antagonism of Beclin 1dependent autophagy by $\mathrm{BCL}-2$ at the endoplasmic reticulum requires NAF-1. EMBO J. 29, 606-618 (2010).

69. Rahmani, M. et al. The kinase inhibitor sorafenib induces cell death through a process involving induction of endoplasmic reticulum stress. Mol. Cell Biol. 27 5499-5513 (2007).

70. Pagliarini, V. et al. Proteolysis of Ambra1 during apoptosis has a role in the inhibition of the autophagic pro-survival response. Cell Death Differ. 19, 1495-1504 (2012). 
71. Pyo, J. O. et al. Essential roles of Atg5 and FADD in autophagic cell death: dissection of autophagic cell death into vacuole formation and cell death. J. Biol. Chem. 280, 20722-20729 (2005).

72. Kubota, $\mathrm{C}$. et al. Constitutive reactive oxygen species generation from autophagosome/lysosome in neuronal oxidative toxicity. J. Biol. Chem. 285, 667-674 (2010)

73. Button, R. W., Roberts, S. L., Willis, T. L., Hanemann, C. O. \& Luo, S. Accumulation of autophagosomes confers cytotoxicity. J. Biol. Chem. 292, 13599-13614 (2017).

74. Huang, K. et al. Honokiol induces apoptosis and autophagy via the ROS/ERK1/ 2 signaling pathway in human osteosarcoma cells in vitro and in vivo. Cell Death Dis. 9, 157 (2018).

75. Zhu, J. et al. Escin induces caspase-dependent apoptosis and autophagy through the ROS/p38 MAPK signalling pathway in human osteosarcoma cells in vitro and in vivo. Cell Death Dis. 8, e3113 (2017).
76. Xilouri, M., Vogiatzi, T., Vekrellis, K, Park, D. \& Stefanis, L. Abberant alphasynuclein confers toxicity to neurons in part through inhibition of chaperonemediated autophagy. PLoS ONE 4, e5515 (2009).

77. Morimoto, N. et al. Increased autophagy in transgenic mice with a G93A mutant SOD1 gene. Brain Res. 1167, 112-117 (2007).

78. Cao, Y. et al. Autophagy is disrupted in a knock-in mouse model of juvenile neuronal ceroid lipofuscinosis. J. Biol. Chem. 281, 20483-20493 (2006).

79. Kim, C., Lee, J., Ko, Y. U. \& Oh, Y. J. Cyclin-dependent kinase 5-mediated phosphorylation of CHIP promotes the tAIF-dependent death pathway in rotenone-treated cortical neurons. Neurosci. Lett. 662, 295-301 (2018).

80. Hansen, M. B., Nielsen, S. E. \& Berg, K. Re-examination and further development of a precise and rapid dye method for measuring cell growth/cell kill. J. Immunol., Methods 119, 203-210 (1989). 